February 27, 2017

\title{
Dirac-Bergmann Constraints in Physics: Singular Lagrangians, Hamiltonian Constraints and the Second Noether Theorem
}

\author{
Luca Lusanna \\ Sezione INFN di Firenze \\ Polo Scientifico \\ Via Sansone 1 \\ 50019 Sesto Fiorentino (FI), Italy \\ E-mail: lusanna@fi.infn.it
}

\begin{abstract}
There is a review of the main mathematical properties of system described by singular Lagrangians and requiring Dirac-Bergmann theory of constraints at the Hamiltonian level. The following aspects are discussed:

i) the connection of the rank and eigenvalues of the Hessian matrix in the Eulerr-Lagrange equations with the chains of first and second class constraints;

ii) the connection of the Noether identities of the second Noether theorem with the Hamiltonian constraints;

iii) the Shanmugadhasan canonical transformation for the identification of the gauge variables and for the search of the Dirac observables, i.e. the quantities invariant under Hamiltonian gauge transformations.

Review paper for a chapter of a future book.
\end{abstract}




\section{INTRODUCTION}

Most of the relevant interactions in physics are described by singular Lagrangians implying the presence of Dirac-Bergmann constraints [1-3] at the Hamiltonian level. This happens for electro-magnetism, for the standard model of particle physics $(S U(3) \times S U(2) \times U(1)$ Yang-Mills fields) and its extensions, for Einstein theory of gravity and for all its generally covariant variants. Also the description of relativistic classical and quantum point particles, needed for bound states in the particle approximation of quantum field theory (QFT), requires Hamiltonian constraints for the elimination of relative times (no time-like excitation is seen in spectroscopy; see the review in Ref.[4] and its bibliography).

In all these theories the main problem at the classical level is the identification of the gauge-invariant physical degrees of freedom, the so called Dirac observables (DO). Instead the main open problem at the quantum level is whether one has to quantize only the DO's or also the gauge variables shifting the search of the physical observables after quantization like in the BRST approach.

In this Chapter I will present a review of the main properties of constrained systems based on my personal viewpoint on the subject at the classical level with some comments on the weak points of the existing quantization approaches. Then in another Chapter I will show their use in special relativity, gauge theories and gravity.

Besides Dirac's book [1] and Ref.[5] I recommend the books in Refs.[6, 7] for an extended treatment of many aspects of the theory also at the quantum level (included the BRST approach). Other books on the subject are in Refs. [8-11]. Instead there is no good treatment of constrained systems in mathematical physics and differential geometry: there are only partial treatments for finite-dimensional systems like presymplectic geometry [12, 13] (see Refs.[14-17] and their bibliography for recent contributions) without any extension to infinite-dimensional systems like field theory [18].

In Section II there is a short review of regular Lagrangian systems for finite-dimensional systems and of their description in the Hamiltonian and velocity space formalisms.

In Section III there is the definition of singular Lagrangians and the description of the Hamiltonian constraints arising in phase space. After the introduction of the Dirac multipliers there is the formulation of the Dirac algorithm for finding the final constraint manifold. After the definition of first and second class constraints there is the diagonalization of the Dirac algorithm. Then the notion of Dirac brackets is introduced for the determination of a phase space without second class constraints.

In Section IV there is the study of the Hamiltonian gauge transformations generated by the first-class constraints. Then the gauge invariant quantities, named Dirac observables (DO), are defined and the reduced phase space is defined. Then the Shanmughadasan canonical transformations for the determination of canonical bases containing a set of DO's for the physical degrees of freedom are described.

In Section $\mathrm{V}$ there is the study of the eigenvalues of the Hessian matrix and of the EulerLagrange (EL) equations when the rank of the Hessian matrix is constant. Then there is a sketch of the pathologies which can appear when such a rank is not constant: proliferation of constraints, ramification of constraint chains, third and fourth class constraints...

In Section VI, after a review of the first Noether theorem and of its extensions there is the description of the second Noether theorem for singular Lagrangians. It is shown which is the connection of the resulting Noether identities with the constraints of the Dirac algorithm. 
In Section VII there is the extension to field theory of constraint theory.

Some open problems are described in the Conclusions.

\section{REGULAR LAGRANGIANS FOR FINITE-DIMENSIONAL SYSTEMS}

Let us consider a finite-dimensional system whose configuration space $Q$ is $n$-dimensional (either $Q=R^{n}$ or $Q$ is an $n$-dimensional manifold with or without boundary), spanned by the configurational coordinates $q^{i}, i=1, . ., n$. We shall give a short review of standard classical mechanics for such systems [19-22].

\section{A. The Second Order Lagrangian Formalism.}

Let the system be described by a time-independent Lagrangian $L(q(t), \dot{q}(t))$, where $q^{i}(t)$ is a curve in $Q$ with time as parameter and $\dot{q}^{i}(t)=\frac{d q^{i}(t)}{d t}$ are the velocities, and by the Lagrangian action $S=\int_{t_{i}}^{t_{f}} d t L(q, \dot{q})$.

The stationarity of the action, $\delta S=\int d t \frac{\delta S}{\delta q^{i}(t)} \delta q^{i}(t)=0$, under variations $\delta q^{i}(t)\left[\delta \dot{q}^{i}=\right.$ $\left.\frac{d}{d t} \delta q^{i}\right]$ which vanish at the endpoints $t_{i}, t_{f}$, identifies the classical motions of the system as those trajectories $q^{i}(t)$ which satisfy the Euler-Lagrange (EL) equations (the summation convention over repeated indices is used; the symbol $\stackrel{\circ}{=}$ denotes an equality which holds only on the trajectories solution of the equations of motion)

$$
\begin{gathered}
L_{i}=\frac{\partial L}{\partial q^{i}}-\frac{d}{d t} \frac{\partial L}{\partial \dot{q}^{i}}=-\left(A_{i j} \ddot{q}^{j}-\alpha_{i}\right) \stackrel{\circ}{=} 0, \\
\alpha_{i}(q, \dot{q})=\frac{\partial L}{\partial q^{i}}-\frac{\partial^{2} L}{\dot{q}^{i} \partial q^{j}} \dot{q}^{j}=\left(1-\dot{q}^{k} \frac{\partial}{\partial \dot{q}^{k}}\right) \frac{\partial L}{\partial q^{i}}-R_{i j} \dot{q}^{j}, \\
R_{i j}(q, \dot{q})=-R_{j i}=\frac{\partial^{2} L}{\partial \dot{q}^{i} \partial q^{j}}-\frac{\partial^{2} L}{\partial \dot{q}^{j} \partial q^{i}} .
\end{gathered}
$$

The Hessian matrix is $A_{i j}(q, \dot{q})=A_{j i}=\frac{\partial^{2} L}{\partial \dot{q}^{i} \partial \dot{q}^{j}}$ and the Lagrangian is said regular when $\operatorname{det} A \neq 0$. If we denote $B=A^{-1}$ the inverse Hessian matrix, it follows that the EL equations can be put in the following normal form $\ddot{q}^{i}-\Lambda^{i} \stackrel{\circ}{=} 0, \Lambda^{i}=B^{i j} \alpha_{j}$.

\section{B. The First Order Hamiltonian Formalism.}

The canonical momenta are defined by $p_{i}=\frac{\partial L(q, \dot{q})}{\partial \dot{q}^{i}}=\mathcal{P}_{i}(q, \dot{q})$ and the regularity condition $\operatorname{det} A \neq 0$ implies that these equations can be inverted to express the velocities $\dot{q}^{i}$ in terms of $q^{k}$ and $p_{k}$.

By means of the Legendre transformation we can re-formulate the second order Lagrangian formalism in the first order Hamiltonian one on the phase space $T^{*} Q$ (the co-tangent bundle) over $Q$ with coordinates $q^{i}, p_{i}$. The Hamiltonian of the system is $\bar{H}=p_{i} \dot{q}^{i}-L$ (we shall denote $\bar{f}=\bar{f}(q, p)$ the functions on phase space) and the phase space action is $\bar{S}=\int_{t_{i}}^{t_{f}} d t \bar{L}$ with $\bar{L}=p_{i} \dot{q}^{i}-\bar{H}$. By asking the stationarity, $\delta \bar{S}=0$, of this action under variations $\bar{\delta} q^{i}$ which vanish at the endpoints $t_{i}, t_{f}$, and under arbitrary variations $\overline{\delta p}_{i}$, we get the first order differential Hamilton equations of motions $\bar{L}_{q i}=\dot{q}^{i}-\frac{\partial \bar{H}}{\partial p_{i}} \stackrel{\circ}{=}$, 
$\bar{L}_{p i}=\dot{p}_{i}+\frac{\partial \bar{H}}{\partial q^{i}} \stackrel{\circ}{=} 0$. The first half of Hamilton equations, ${\overline{L_{q i}}}^{-} \stackrel{\circ}{=}$, have a purely kinematical content: they give the inversion of the equations $p_{i}=\mathcal{P}_{i}(q, \dot{q})$, i.e. $\dot{q}^{i}=\bar{g}^{i}(q, p)$.

By introducing the Poisson brackets ${ }^{1}\{\bar{A}(q, p), \bar{B}(q, p)\}=\frac{\partial \bar{A}}{\partial q^{i}} \frac{\partial \bar{B}}{\partial p^{i}}-\frac{\partial \bar{A}}{\partial p^{i}} \frac{\partial \bar{B}}{\partial q^{i}}$, we can rewrite the Hamilton equations in the form $\dot{q}^{i} \stackrel{\varrho}{=}\left\{q^{i}, \bar{H}\right\}=\bar{X}_{\bar{H}} q^{i}, \dot{p}_{i} \stackrel{\circ}{=}\left\{p_{i}, \bar{H}\right\}=\bar{X}_{\bar{H}} p_{i}$, where we introduced the evolution Hamiltonian vector field $\bar{X}_{\bar{H}}=\{., \bar{H}\}$.

In the regular case every function $f(q, \dot{q})$ is projectable to phase space: $f(q, \dot{q})=\bar{f}(q, p)$ by using $\dot{q}^{i}=\bar{g}^{i}(q, p)$.

Let us remark that there are two intrinsic formulations of the Hamiltonian description (we consider only the case of an exact symplectic structure arising when there is a well defined Lagrangian):

i) a time-independent one on the symplectic manifold $T^{*} Q$ (the symplectic structure) based on the Cartan-Liouville one-form $\bar{\theta}=p_{i} d q^{i}$ and on the closed symplectic two-form $\bar{\omega}=d \bar{\theta}=$ $d p_{i} \wedge d q^{i}, d \bar{\omega}=0$, where we gave the coordinate expression in Darboux coordinates adapted to the symplectic structure; ii) a time-dependent one on $R \times T^{*} Q$ (the contact structure; $R$ is the time axis; this formulation allows one to treat also time-dependent Lagrangians, $L(t, q, \dot{q}))$ based on the Poincare'-Cartan one-form $\tilde{\bar{\theta}}=\bar{L} d t=\bar{\theta}-\bar{H} d t$ and on the closed contact two-form $\tilde{\bar{\omega}}=d \tilde{\bar{\theta}}=\bar{\omega}-d \bar{H} \wedge d t$.

In the regular case all these descriptions are equivalent.

\section{The First Order Velocity Space Formalism.}

When the second order differential equations of motion are in the normal form $\left(\ddot{q}^{i} \stackrel{\circ}{=} \Lambda^{i}\right)$, they can be re-written as a set of first order differential equations on the velocity space $T Q$ (the tangent bundle) over $\mathrm{Q}$ with coordinates $q^{i}, v^{i}$ (we shall denote $\tilde{f}=\tilde{f}(q, v)$ the functions on the velocity space and we have $\left.f(q, \dot{q})=\left.\tilde{f}(q, v)\right|_{v=\dot{q}}\right)$ with the following position

$$
\dot{q}^{i} \stackrel{\circ}{=} v^{i}, \quad \dot{v}^{i} \stackrel{\circ}{=} \tilde{\Lambda}^{i}(q, v) .
$$

By introducing $\tilde{L}(q, v)=\left.L(q, \dot{q})\right|_{\dot{q}=v}$ and the energy function $\tilde{E}=\frac{\partial \tilde{L}}{\partial v^{i}} v^{i}-\tilde{L}$, we can define the $T Q$ action $\tilde{S}=\int_{t_{i}}^{t_{f}} d t \tilde{L}_{v}$ and Lagrangian $\tilde{L}_{v}=\frac{\partial \tilde{L}}{\partial v^{i}} \dot{q}^{i}-\tilde{E}$, whose stationarity yields the velocity space first order differential equations of motion $\left(\tilde{A}_{i j}, \tilde{B}^{i j}, \tilde{R}_{i j}\right.$ are the $T Q$ expressions of $A_{i j}, B^{i j}, R_{i j}$ respectively)

$$
\begin{aligned}
& \tilde{L}_{q i}=-\tilde{A}_{i j}\left[\dot{v}^{j}+\tilde{B}^{j k}\left(\frac{\partial \tilde{E}}{\partial q^{k}}+\tilde{R}_{k h} \tilde{B}^{h r} \frac{\partial \tilde{E}}{\partial v^{r}}\right)\right]-\tilde{R}_{i j}\left(\dot{q}^{j}-v^{j}\right) \stackrel{\circ}{=}, \\
& \tilde{L}_{v i}=\tilde{A}_{i j}\left(\dot{q}^{j}-v^{j}\right) \stackrel{\circ}{\circ} .
\end{aligned}
$$

The normal form of these equations are Eqs.(2.2), which can also be written in the form $\dot{q}^{i} \stackrel{\circ}{=} v^{i}=\left\{q^{i}, \tilde{E}\right\}_{L}, \dot{v}^{i} \stackrel{\circ}{=}-\tilde{B}^{i j}\left(\frac{\partial \tilde{E}}{\partial q^{j}}+\tilde{R}_{j k} \tilde{B}^{k h} \frac{\partial \tilde{E}}{\partial v^{h}}\right)=\tilde{B}^{i j} \tilde{\alpha}_{j}=\tilde{\Lambda}^{i}=\left\{v^{i}, \tilde{E}\right\}_{L}$, where we

${ }^{1}$ They satisfy: i) $\{\bar{f}, \bar{g}\}=-\{\bar{g}, \bar{f}\}$; ii) $\left\{\bar{f}, \bar{g}_{1} \bar{g}_{2}\right\}=\left\{\bar{f}, \bar{g}_{1}\right\} \bar{g}_{2}+\bar{g}_{1}\left\{\bar{f}, \bar{g}_{2}\right\}$ (Leibnitz rule for derivations); iii) $\{\{\bar{f}, \bar{g}\}, \bar{u}\}+\{\{\bar{g}, \bar{u}\}, \bar{f}\}+\{\{\bar{u}, \bar{f}\}, \bar{g}\}=0$ (Jacobi identity). 
have introduced the (Lagrangian dependent) $T Q$ Poisson brackets $\{\tilde{f}, \tilde{g}\}_{L}=\tilde{B}^{i j}\left(\frac{\partial \tilde{f}}{\partial q^{i}} \frac{\partial \tilde{g}}{\partial v^{j}}-\right.$ $\left.\frac{\partial \tilde{f}}{\partial v^{j}} \frac{\partial \tilde{g}}{\partial q^{i}}\right)-\frac{\partial \tilde{f}}{\partial v^{i}} \tilde{B}^{i h} \tilde{R}_{h k} \tilde{B}^{k j} \frac{\partial \tilde{g}}{\partial v^{j}},\left\{q^{i}, q^{j}\right\}_{L}=0,\left\{q^{i}, v^{j}\right\}_{L}=\tilde{B}^{i j},\left\{v^{i}, v^{j}\right\}_{L}=\tilde{B}^{i h} \tilde{R}_{h k} \tilde{B}^{k j}$.

Therefore we have the same symplectic structure in $T Q$ and $T^{*} Q$. In this context the Legendre transformation is defined as the fiber derivative $F L$ of $\tilde{L}(q, v)$ : it is a linear and fiber preserving mapping from $T Q$ to $T^{*} Q$ defined by $F L:(q, v) \in T_{q} Q \mapsto p_{i} d q^{i} \in T_{q}^{*} Q$ $\left(p_{i}=\frac{\partial \tilde{L}}{\partial v^{i}}\right)$. When $F L$ is a global diffeomorphism of $T Q$, the Lagrangian $\tilde{L}(q, v)$ is said to be hyper-regular and one has a global Hamiltonian formalism (i.e. $\bar{H}(q, p)$, the Legendre transform of $\tilde{E}(q, v)$, exists globally on $\left.T^{*} Q\right)$. When $F L$ is only a local diffeomorphism of $T Q, \tilde{L}(q, v)$ is said to be regular and $\bar{H}(q, p)$ exists only locally. In the regular case $F L$ is a symplectomorphism which connects the symplectic structures of $T^{*} Q$ and $T Q$.

The definition $\dot{q}^{i} \stackrel{\circ}{=} v^{i} \equiv \tilde{\Gamma} q^{i}$ and Eqs.(2.2) imply $\ddot{q}^{i} \stackrel{\circ}{=} \frac{d v^{i}}{d t} \stackrel{\circ}{=} \tilde{\Lambda}^{i}=\tilde{\Gamma} v^{i}$ in the regular case: this is called the second order differential equation (SODE) condition ensuring that $\tilde{\Gamma}$ is a second order vector field. See Ref.[23] for the study of the phase space over the velocity space, i.e. $T^{*}(T Q)$.

\section{SINGULAR LAGRANGIANS AND HAMILTONIAN CONSTRAINTS FOR FINITE-DIMENSIONAL SYSTEMS}

Let us consider a finite-dimensional system with a $n$-dimensional configuration space $Q$ admitting a global coordinate system $q^{i}, i=1, . ., n^{2}$. Let its dynamics be described by a time-independent singular Lagrangian $L(q(t), \dot{q}(t))$ (the extension to the time-dependent case does not introduce further complications), namely such that its Hessian matrix is singular: $\operatorname{det} \frac{\partial^{2} L(q, \dot{q})}{\partial \dot{q}^{i} \partial \dot{q}^{j}}=0$.

In this Section we introduce the Hamiltonian formalism for singular systems and then we shall come back to study the second order Lagrangian formalism, giving also some information about the velocity space formalism in Section $\mathrm{V}$ after having looked at the notion of DO in Section IV. We shall follow Refs. [6, 23-25].

\section{A. Primary Hamiltonian Constraints and the Hamilton-Dirac Equations.}

When the Hessian matrix is singular the EL equations (2.1) cannot be put in normal form. This means that the accelerations $\ddot{q}^{i}$ cannot be uniquely determined in terms of $q^{i}, \dot{q}^{i}$ and that the solutions of the EL equations may depend on arbitrary functions of time.

Moreover $\operatorname{det} A_{i j}(q, \dot{q})=0$ implies that the canonical momenta cannot be inverted to get the velocities $\dot{q}^{i}$ in terms of $q^{i}, p_{i}$. The $n$ functions $p_{i}=\mathcal{P}_{i}(q, \dot{q})$ are not functionally independent, namely there are as many identities $\phi_{A}(q, \mathcal{P}(q, \dot{q})) \equiv 0, \mathrm{~A}=1, . ., \mathrm{m}$, as null eigenvalues of the Hessian matrix. In phase space $\left(T^{*} Q\right)$ these identities become the primary Hamiltonian constraints (their functional form is highly arbitrary)

$$
\bar{\phi}_{A}(q, p)=0, \quad A=1, . ., m,
$$

which identify the region $\gamma$ of $T^{*} Q$ allowed to the configurations of the singular system. Points outside $\gamma$ are not accessible, but we go on to work in $T^{*} Q$ to utilize its symplectic

\footnotetext{
${ }^{2}$ Otherwise the following treatment will only hold locally in a chart of the coordinate atlas of $Q$.
} 
structure ( $\gamma$ in general has not such a structure), i.e. its Poisson brackets.

Eq.(3.1) is usually written with Dirac's weak equality sign $\approx$, i.e. $\bar{\phi}_{A}(q, p) \approx 0, \mathrm{~A}=1, . ., \mathrm{m}$. An equation $\bar{f}(q, p) \approx 0$ means that the function $\bar{f}$ vanishes on $\gamma$, but can be different from zero outside $\gamma$ so that it cannot be put equal to zero inside the $T^{*} Q$ Poisson brackets even when they are restricted to $\gamma$. Instead the strong equality symbol $\equiv$ (like for identical) is used for a function $\bar{f}(q, p)$ vanishing on $\gamma, \bar{f} \approx 0$, and such that also its differential vanishes on $\gamma, d \bar{f} \approx 0$; such a function (for instance $\bar{f}=\bar{\phi}_{A}^{2}$ ) can be put equal to zero inside Poisson brackets restricted to $\gamma$.

Let us assume that the rank of the Hessian matrix $A_{i j}(q, \dot{q})$ is constant and equal to $m$ for every value of $(q, \dot{q})$. See Section V and Ref.[24] for what may happen when we relax this assumption.

Let us also assume that the singular Lagrangian is such that the region $\gamma$ defined by the primary constraints is a $(2 n-m)$-dimensional sub-manifold of $T^{*} Q$ ( $\gamma$ is the primary constraint sub-manifold). While $p_{k} \approx 0$ is an acceptable constraint, neither $p_{k}^{2} \approx 0$ nor $\sqrt{p_{k}} \approx 0$ are acceptable. When the rank of the Hessian matrix is not constant (see Section $\mathrm{V})$, constraints of the type $p_{k}^{2} \approx 0$ may appear. Constraints of the type $\left(p_{k}\right)^{2}+\left(q^{h}\right)^{2} \approx 0$ must be put in the form $p_{k} \approx 0$ and $q^{h} \approx 0$.

While in the regular case the invertibility of the equations $p_{i}=\mathcal{P}(q, \dot{q})$ to $\dot{q}^{i}=\bar{g}^{i}(q, p)$ implies that all the velocities $\dot{q}^{i}$ are projectable to $T^{*} Q$, now there will be $m$ independent (but with a not uniquely determined functional form) functions of the velocities $g^{A}(q, \dot{q})$ (named non-projectable velocity functions) not projectable to $T^{*} Q$.

Let us also assume that the singular Lagrangian admits a well defined Legendre transformation. Then, if we introduce the function $H_{c}(q, \dot{q})=p_{i} \dot{q}^{i}-L(q, \dot{q})=\mathcal{P}_{i}(q, \dot{q}) \dot{q}^{i}-L(q, \dot{q})$, we get $\delta H_{c}=\delta p_{i} \dot{q}^{i}+p_{i} \delta \dot{q}^{i}-\frac{\partial L}{\partial q^{i}} \delta q^{i}-\frac{\partial L}{\partial \dot{q}^{i}} \delta \dot{q}^{i}=\dot{q}^{i} \delta p_{i}-\frac{\partial L}{\partial q^{i}} \delta q^{i}=\delta \bar{H}_{c}$ as in the regular case. This means that $H_{c}(q, \dot{q})$ is projectable to a well defined canonical Hamiltonian also in the singular case

$$
H_{c}(q, \dot{q})=\bar{H}_{c}(q, p), \quad\left(\frac{\partial \bar{H}_{c}}{\partial q^{i}}+\frac{\partial L}{\partial q^{i}}\right) \delta q^{i}+\left(\frac{\partial \bar{H}_{c}}{\partial p_{i}}-\dot{q}^{i}\right) \delta p_{i}=0 .
$$

But in the singular case Eqs.(3.2) are meaningful only if $\left(\delta q^{i}, \delta p_{i}\right)$ is a vector tangent to the primary constraint sub-manifold $\gamma$, so that one gets (see Ref.[6] for a demonstration)

$$
\begin{aligned}
& \dot{q}^{i}=\frac{\partial \bar{H}_{c}}{\partial p_{i}}+u^{A} \frac{\partial \bar{\phi}_{A}}{\partial p_{i}}=\left\{q^{i}, \bar{H}_{c}\right\}+u^{A}\left\{q^{i}, \bar{\phi}_{A}\right\} \\
& \dot{p}_{i}=\left.\frac{\partial L}{\partial q^{i}}\right|_{\dot{q}}=-\frac{\partial \bar{H}_{c}}{\partial q^{i}}-u^{A} \frac{\partial \bar{\phi}_{A}}{\partial q^{i}}=\left\{p_{i}, \bar{H}_{c}\right\}+u^{A}\left\{p_{i}, \bar{\phi}_{A}\right\}
\end{aligned}
$$

where the EL equations have been used in the second line. Since the velocities are not projectable to $T^{*} Q$ in the singular case, the functions $u^{A}$ in the first line of Eqs.(3.3) cannot be functions on $T^{*} Q$ but must depend also on the velocities: $u^{A}=u^{A}(q, p, \dot{q})=$ $u^{A}(q, \mathcal{P}(q, \dot{q}), \dot{q})=v^{A}(q, \dot{q})$. These multipliers identify a canonical functional form $g_{(u)}^{A}=v^{A}$ of the non-projectable velocity functions $g^{A}(q, \dot{q})$.

However, since Eqs.(3.3) are the Hamilton equations for the singular case (the so called Hamilton-Dirac equations), their right side cannot depend explicitly on the velocities. Therefore a consistent Hamiltonian formalism is obtained by replacing the functions $u^{A}=v^{A}(q, \dot{q})$ 
with arbitrary multipliers $\lambda^{A}(t)$ (the so called Dirac multipliers), by introducing the Dirac Hamiltonian

$$
\bar{H}_{D}(q, p, \lambda)=\bar{H}_{c}(q, p)+\lambda^{A}(t) \bar{\phi}_{A}(q, p),
$$

and by introducing the $T^{*} Q$ action $\bar{S}=\int_{t_{i}}^{t_{f}} d t\left(p_{i} \dot{q}^{i}-\bar{H}_{c}-\lambda^{A}(t) \bar{\phi}_{A}\right)$.

In it $q^{i}, p_{i}$ and $\lambda^{A}$ are considered as independent variables. The stationarity, $\delta \bar{S}=0$, under variations $\delta q^{i}, \delta p_{i}, \delta \lambda^{A}$ with the only restriction $\delta q^{i}\left(t_{f}\right)=\delta q^{i}\left(t_{i}\right)=0$ yields the Hamilton-Dirac equations supplemented by the definition of the primary constraint submanifold

$$
\begin{aligned}
\dot{q}^{i} & \stackrel{\circ}{=}\left\{q^{i}, \bar{H}_{D}(q, p, \lambda)\right\}, \quad \text { or } \quad \bar{L}_{D q}^{i}=\dot{q}^{i}-\left\{q^{i}, \bar{H}_{D}\right\} \stackrel{\circ}{=} 0, \\
\dot{p}_{i} & \stackrel{\circ}{=}\left\{p_{i}, \bar{H}_{D}(q, p, \lambda)\right\}, \quad \text { or } \quad \bar{L}_{D p i}=\dot{p}_{i}-\left\{p_{i}, \bar{H}_{D}\right\} \stackrel{\circ}{=} 0, \\
\bar{\phi}_{A}(q, p) & \stackrel{\circ}{=} 0 .
\end{aligned}
$$

The kinematical equations $\dot{q}^{i} \stackrel{\circ}{=}\left\{q^{i}, \bar{H}_{D}\right\}, \bar{\phi}_{A} \stackrel{\circ}{=}$, determine the canonical momenta and the Dirac multipliers in terms of the coordinates and momenta (if suitable regularity conditions hold), namely we get: i) $p_{i}=\mathcal{P}_{i}(q, \dot{q})$; ii) the canonical functional form $g_{(\lambda)}^{A}$ of the nonprojectable velocity functions $g^{A}(q, \dot{q})$ associated with the chosen functional form of the primary constraints as the $(q, \dot{q})$ space expression of the Dirac multipliers $g_{(\lambda)}^{A}(q, \dot{q}) \stackrel{\circ}{=} \lambda^{A}(t)$. Then we can make the inverse Legendre transformation and recover the original singular Lagrangian: $p_{i} \dot{q}^{i}-\bar{H}_{D} \stackrel{\circ}{=} \mathcal{P}_{i}(q, \dot{q}) \dot{q}^{i}-\bar{H}_{c}(q, \mathcal{P}(q, \dot{q}))=L(q, \dot{q})$.

\section{B. Dirac's Algorithm for the Determination of the Final Constraint Sub-manifold.}

The inspection of the functional form of the canonical momenta $p_{i}=\mathcal{P}_{i}(q, \dot{q})$ identifies the primary constraint sub-manifold $\gamma \subset T^{*} Q$. The Hamiltonian formalism will produce a consistent treatment of singular systems only if $\gamma$ does not change with time, namely if the primary constraints $\bar{\phi}_{A}(q, p) \approx 0$ are constant of motion with respect to the evolution generated by the Dirac Hamiltonian

$$
\begin{aligned}
\frac{d \bar{\phi}_{A}(q, p)}{d t} & \stackrel{\circ}{=}\left\{\bar{\phi}_{A}(q, p), \bar{H}_{D}\right\}= \\
& =\left\{\bar{\phi}_{A}(q, p), \bar{H}_{c}(q, p)\right\}+\lambda^{B}(t)\left\{\bar{\phi}_{A}(q, p), \bar{\phi}_{B}(q, p)\right\} \approx 0 \quad \text { on } \gamma, A=1, \ldots, m .
\end{aligned}
$$

Some of these equations may be void $(0=0)$. The non-void ones, restricted to $\gamma$, have to be separated in two disjoint groups:

i) a set of $m_{1} \leq m$ equations independent from the Dirac multipliers

$$
\bar{\chi}_{a_{1}}^{(1)}(q, p) \approx 0 \quad \text { on } \gamma, \quad a_{1}=1, . ., m_{1} ;
$$

ii) a set of $h_{1}$ equations $\left(h_{1} \leq m, h_{1}+m_{1} \leq m\right)$ for the Dirac multipliers 


$$
\bar{f}_{\tilde{A}_{1} B}(q, p) \lambda^{B}(t)+\bar{g}_{\tilde{A}_{1}}(q, p) \approx 0 \quad \text { on } \gamma, \quad \tilde{A}_{1}=1, . ., h_{1},
$$

with $\bar{f}_{\tilde{A}_{1}}=\bar{h}_{\tilde{A}_{1}}^{A}\left\{\bar{\phi}_{A}, \bar{\phi}_{B}\right\}, \bar{g}_{\tilde{A}_{1}}=\bar{h}_{\tilde{A}_{1}}^{A}\left\{\bar{\phi}_{A}, \bar{H}_{c}\right\}$ for some functions $\bar{h}_{\tilde{A}_{1}}^{A}$.

Let us remark that without certain regularity conditions on the singular Lagrangian this separation cannot be done in a unique way: i) we can get different separations in different regions of $\gamma$; ii) also in the same point of $\gamma$ we can have alternative inequivalent separations. A regularity condition which eliminates most (if not all) of these possibilities is that the anti-symmetric matrix $\bar{M}_{A B}(q, p)=\left(\left\{\bar{\phi}_{A}(q, p), \bar{\phi}_{B}(q, p)\right\}\right)$ of the Poisson brackets of the primary constraints has constant rank on $\gamma$.

Let us assume that there is a unique separation given by Eqs. (3.7), (3.8). Eqs. (3.7) are called secondary constraints and define a secondary constraint sub-manifold $\gamma_{1}$ of $\gamma$ to which the description of the singular system has to be restricted for consistency. Differently from the primary constraints, the secondary constraints are defined by using the equations of motion. Eqs.(3.8) show that on the constraint sub-manifold $\gamma_{1} \subset \gamma \subset T^{*} Q$ there may be less arbitrariness than on $\gamma$, because $h_{1} \leq m$ Dirac multipliers $\lambda^{A}(t)$ are determined by these equations and an equal number of velocity functions $g_{(\lambda)}^{A}(q, \dot{q})$, not projectable onto $\gamma$, become projectable onto $\gamma_{1} \subset \gamma$.

If $\bar{U}^{A}(q, p)$ is a particular solution of the in-homogeneous Eqs.(3.8) and $\bar{V}_{A_{1}}^{A}(q, p), A_{1}=$ $1, \ldots, k_{1}=m-h_{1}$, are independent solutions of the homogeneous equations $\bar{f}_{\tilde{A}_{1} B} \lambda^{B}(t)=$ $\bar{h}_{\tilde{A}_{1}}^{A}\left\{\bar{\phi}_{A}, \bar{\phi}_{B}\right\} \lambda^{B}(t)=0$ (namely $\left\{\bar{\phi}_{A}, \bar{\phi}_{B}\right\} \bar{V}_{A_{1}}^{B} \equiv 0$ ), then the general solution of Eqs.(3.8) is

$$
\lambda^{A}(t) \approx \bar{U}^{A}(q, p)+\lambda^{(1) A_{1}}(t) \bar{V}_{A_{1}}^{A}(q, p) \quad \text { on } \gamma_{1}, \quad A_{1}=1, . ., k_{1}=m-h_{1},
$$

with the $\lambda^{(1) A_{1}}(t)$ 's being new $k_{1}=m-h_{1}$ arbitrary Dirac multipliers.

The Dirac Hamiltonian on $\gamma_{1} \subset \gamma$ is

$$
\begin{aligned}
\bar{H}_{D}(q, p, \lambda) & =\bar{H}_{c}^{(1)}(q, p)+\lambda^{(1) A_{1}}(t) \bar{\phi}_{A_{1}}^{(1)}(q, p) \quad \text { on } \gamma_{1}, \\
\bar{\phi}_{A_{1}}^{(1)}(q, p) & =\bar{V}_{A_{1}}^{A}(q, p) \bar{\phi}_{A}(q, p), \quad A_{1}=1, . ., k_{1}=m-h_{1}, \\
\bar{H}_{c}^{(1)}(q, p) & =\bar{H}_{c}(q, p)+\bar{U}^{A}(q, p) \bar{\phi}_{A}(q, p) \approx \bar{H}_{c}(q, p) \quad \text { on } \gamma_{1} .
\end{aligned}
$$

The remaining Dirac multipliers $\lambda^{(1) A_{1}}(t)$ are now multiplied by the linear combinations $\bar{\phi}_{A_{1}}^{(1)}(q, p)$ of the original primary constraints.

When there are secondary constraints $\bar{\chi}_{a_{1}}^{(1)}(q, p) \approx 0$, for consistency we must ask that the secondary constraint sub-manifold $\gamma_{1} \subset \gamma$ does not change with time: the secondary constraints must be constants of motion on $\gamma_{1}$ with respect to the Dirac Hamiltonian

$$
\begin{aligned}
& \left.\frac{d \bar{\chi}_{a_{1}}^{(1)}(q, p)}{d t} \stackrel{\circ}{=} \bar{\chi}_{a_{1}}^{(1)}(q, p), \bar{H}_{D}(q, p, \lambda)\right\} \approx \\
& \approx\left\{\bar{\chi}_{a_{1}}^{(1)}(q, p), \bar{H}_{c}^{(1)}(q, p)\right\}+\lambda^{(1) A_{1}}(t)\left\{\bar{\chi}_{a_{1}}^{(1)}(q, p), \bar{\phi}_{A_{1}}^{(1)}(q, p)\right\} \approx 0 \quad \text { on } \gamma_{1} \text {, } \\
& A_{1}=1, . ., k_{1}=m-h_{1}, \quad a_{1}=1, . ., m_{1} .
\end{aligned}
$$

By assuming the regularity condition that the rank of the matrix $\left(\left\{\bar{\chi}_{a_{1}}^{(1)}, \bar{\phi}_{A_{1}}^{(1)}\right\}\right)$ is constant on $\gamma_{1}$, the non-void Eqs.(3.11) may be separated in the two disjoint sets 


$$
\begin{gathered}
\text { i) } \bar{\chi}_{a_{2}}^{(2)}(q, p) \approx 0 \quad \text { on } \gamma_{1}, \quad a_{2}=1, . ., m_{2} \leq m_{1} \\
\text { ii) } \bar{f}_{\tilde{A}_{2} B_{1}}(q, p) \lambda^{(1) B_{1}}(t)+\bar{g}_{\tilde{A}_{2}}(q, p) \approx 0 \quad \text { on } \gamma_{1}, \quad \tilde{A}_{2}=1, . ., h_{2} \leq k_{1} .
\end{gathered}
$$

Eqs.(3.12) are the tertiary constraints and define a new constraint sub-manifold $\gamma_{2} \subset \gamma_{1}$. With the same procedure delineated above we arrive at the conclusion that only on $\gamma_{2}$ can there be a consistent dynamics for the singular system with (in general) a reduction of the number of independent Dirac multipliers, which are replaced by the new ones $\lambda^{(2) A_{2}}(t)$ due to Eqs.(3.13). The dynamics is described in terms of the following quantities

$$
\begin{aligned}
\lambda^{(1) A_{1}}(t) & \approx \bar{U}^{(1) A_{1}}(q, p)+\lambda^{(2) A_{2}}(t) \bar{V}_{A_{2}}^{(1) A_{1}}(q, p) \quad \text { on } \gamma_{2}, \quad A_{2}=1, . ., k_{2}=m-h_{1}-h_{2}, \\
\bar{H}_{D}(q, p, \lambda) & =\bar{H}_{c}^{(2)}(q, p)+\lambda^{(2) A_{2}}(t) \bar{\phi}_{A_{2}}^{(2)}(q, p), \quad \text { on } \gamma_{2}, \\
\bar{\phi}_{A_{2}}^{(2)}(q, p) & =\bar{V}_{A_{2}}^{(1) A_{1}}(q, p) \bar{\phi}_{A_{1}}^{(1)}(q, p)=\bar{V}_{A_{2}}^{(1) A_{1}}(q, p) \bar{V}_{A_{1}}^{A}(q, p) \bar{\phi}_{A}(q, p), \\
\bar{H}_{c}^{(2)}(q, p) & =\bar{H}_{c}^{(1)}(q, p)+\bar{U}^{(1) A_{1}}(q, p) \bar{\phi}_{A_{1}}^{(1)}(q, p)= \\
& =\bar{H}_{c}(q, p)+\left(\bar{U}^{A}(q, p)+\bar{U}^{(1) A_{1}}(q, p) \bar{V}_{A_{1}}^{A}(q, p)\right) \bar{\phi}_{A}(q, p) .
\end{aligned}
$$

This procedure is iterated till a final stage $(f)$ in which the final constraint sub-manifold $\bar{\gamma}=\gamma_{f} \subset \ldots \subset \gamma_{1} \subset \gamma \subset T^{*} Q$ is determined by $(f+1)$-ary constraints

$$
\bar{\chi}_{a_{f}}^{(f)}(q, p) \approx 0 \quad \text { on } \gamma_{f-1}, \quad a_{f}=1, . ., m_{f} \leq m_{f-1} \leq \ldots \leq m .
$$

On $\bar{\gamma}=\gamma_{f}$ we have

$$
\begin{aligned}
& \bar{H}_{D}(q, p, \lambda)=\bar{H}_{c}^{(f)}(q, p)+\lambda^{(f) A_{f}}(t) \bar{\phi}_{A_{f}}^{(f)}(q, p), \quad \text { on } \gamma_{f}, \\
& \bar{\phi}_{A_{f}}^{(f)}(q, p)=\bar{V}_{A_{f}}^{(f-1)} A_{f-1}(q, p) \ldots \bar{V}_{A_{1}}^{A}(q, p) \bar{\phi}_{A}(q, p), \quad A_{f}=1, . ., k_{f}=m-h_{1}-. .-h_{f}, \\
& \bar{H}_{c}^{(f)}(q, p)=\bar{H}_{c}(q, p)+\left(\bar{U}^{A}(q, p)+\bar{U}^{(1) A_{1}}(q, p) \bar{V}_{A_{1}}^{A}(q, p)+\ldots+\right. \\
& \left.+\bar{U}^{(f-1) A_{f-1}}(q, p) \bar{V}_{A_{f-1}(f-2)}^{A_{f-2}}(q, p) \ldots \bar{V}_{A_{1}}^{A}(q, p)\right) \bar{\phi}_{A}(q, p) \approx \bar{H}_{c}(q, p), \\
& \left.\frac{d \bar{\chi}_{a_{f}}^{(f)}(q, p)}{d t} \stackrel{\circ}{=} \bar{\chi}_{a_{f}}^{(f)}(q, p), \bar{H}_{D}(q, p, \lambda)\right\} \quad \text { identically satisfied }
\end{aligned}
$$

with only $k_{f}=m-h_{1}-. .-h_{f}$ independent final Dirac multipliers $\lambda^{(f) A_{f}}(t)$. Only an equal number of velocity functions $g_{(\lambda)}^{A}(q, \dot{q})$ cannot be projected onto $\bar{\gamma}=\gamma_{f} \subset T^{*} Q$.

The solutions of the Hamilton-Dirac equations on $\bar{\gamma}$ will depend on the $k_{f}$ arbitrary functions of time $\lambda^{(f) A_{f}}(t)$, which describe the non-deterministic aspects of the time evolution of the singular system. 


\section{First and Second Class Constraints.}

The final constraint manifold $\bar{\gamma}=\gamma_{f} \subset . . \subset \gamma \subset T^{*} Q$ is determined by the full set of primary, secondary,.. constraints $\bar{\phi}_{A}(q, p) \approx 0(A=1, . ., m), \bar{\chi}_{a_{1}}^{(1)}(q, p) \approx 0\left(a_{1}=1, . ., m_{1}\right)$, $\ldots, \bar{\chi}_{a_{f}}^{(f)}(q, p) \approx 0\left(a_{f}=1, . ., m_{f}\right)$. Let us denote all the $M=m+m_{1}+. .+m_{f}$ constraints with the collective notation

$$
\bar{\zeta}_{\mathcal{A}}(q, p) \approx 0, \quad \mathcal{A}=1, . ., M
$$

because the property of being a primary, secondary .. constraint is not important.

Let us also denote with $\lambda^{\bar{A}}(t)$ and $\bar{\phi}_{\bar{A}}(q, p)$ with $\bar{A}=1, . ., \bar{k}=m-h_{1}-. .-h_{f}$ the final arbitrary Dirac multipliers and the associated linear combinations of primary constraints respectively, so that the Dirac Hamiltonian on $\bar{\gamma}$ is

$$
\begin{aligned}
& \bar{H}_{D}(q, p, \lambda)=\bar{H}_{c}^{(F)}(q, p)+\lambda^{\bar{A}}(t) \bar{\phi}_{\bar{A}}(q, p), \quad \text { on } \bar{\gamma}, \\
& \bar{\phi}_{\bar{A}}(q, p)=\bar{V}^{(f-1)}{ }_{\bar{A}}^{A_{f-1}}(q, p) \ldots \bar{V}_{A_{1}}^{A}(q, p) \bar{\phi}_{A}(q, p), \quad \bar{A}=1, . ., \bar{k}, \\
& \bar{H}_{c}^{(F)}(q, p)=\bar{H}_{c}(q, p)+\bar{U}^{(F) A}(q, p) \bar{\phi}_{A}(q, p) \approx \bar{H}_{c}(q, p), \\
& \bar{U}^{(F) A}(q, p)=\bar{U}^{A}(q, p)+\bar{U}^{(1) A_{1}}(q, p) \bar{V}_{A_{1}}^{A}(q, p)+\ldots+ \\
& +\bar{U}^{(f-1) A_{f-1}}(q, p) \bar{V}^{(f-2)}{ }_{A_{f-1}}^{A_{f-2}}(q, p) \ldots \bar{V}_{A_{1}}^{A}(q, p) .
\end{aligned}
$$

As a result of the previous construction all the constraints are preserved in time on $\bar{\gamma}$,

$$
\frac{d \bar{\zeta}_{\mathcal{A}}}{d t} \stackrel{\circ}{=}\left\{\bar{\zeta}_{\mathcal{A}}, \bar{H}_{D}\right\} \approx 0 \Rightarrow \quad\left\{\bar{\zeta}_{\mathcal{A}}, \bar{H}_{c}^{(F)}\right\} \approx 0, \quad\left\{\bar{\zeta}_{\mathcal{A}}, \bar{\phi}_{\bar{A}}\right\} \approx 0
$$

Let us call a first class function a function $\bar{f}(q, p)$ on $T^{*} Q$ whose Poisson brackets with every constraint is weakly zero

$$
\left\{\bar{f}(q, p), \bar{\zeta}_{\mathcal{A}}(q, p)\right\}=\bar{F}_{\mathcal{A}}^{\mathcal{B}}(q, p) \bar{\zeta}_{\mathcal{B}}(q, p) \approx 0 \quad \text { on } \bar{\gamma}
$$

If two functions $\bar{f}, \bar{g}$ are first class, also their Poisson bracket is a first class function due to the Jacobi identity: $\left\{\{\bar{f}, \bar{g}\}, \bar{\zeta}_{\mathcal{A}}\right\}=\left\{\bar{f},\left\{\bar{g}, \bar{\zeta}_{\mathcal{A}}\right\}\right\}-\left\{\bar{g},\left\{\bar{f}_{,} \bar{\zeta}_{\mathcal{A}}\right\}\right\}=\left\{\bar{f}, \bar{G}_{\mathcal{A}}^{\mathcal{B}} \bar{\zeta}_{\mathcal{B}}\right\}-\left\{\bar{g}, \bar{F}_{\mathcal{A}}^{\mathcal{B}} \bar{\zeta}_{\mathcal{B}}\right\}=$ $\bar{K}_{\mathcal{A}}^{\mathcal{B}} \bar{\zeta}_{\mathcal{B}} \approx 0$

All the functions which are not first class are named second class functions.

Eqs.(3.19) show that both the final canonical Hamiltonian $\bar{H}_{c}^{(F)}(q, p)$ and the final combinations $\bar{\phi}_{\bar{A}}(q, p), \bar{A}=1, . ., \bar{k}$, of the primary constraints are first class functions.

It is of fundamental importance in constraint theory to separate the constraints $\bar{\zeta}_{\mathcal{A}}=$ $\left(\bar{\phi}_{A}, \bar{\chi}_{a_{1}}^{(1)}, . ., \bar{\chi}_{a_{f}}^{(f)}\right)$ in two groups: i) the first class constraints $\bar{\Phi}_{(1) \mathcal{A}_{1}}=\bar{k}_{\mathcal{A}_{1}}^{\mathcal{A}} \bar{\zeta}_{\mathcal{A}} \approx 0$, $\mathcal{A}_{1}=1, . ., r_{1},\left\{\bar{\Phi}_{(1) \mathcal{A}_{1}}, \bar{\zeta}_{\mathcal{A}}\right\} \approx 0$; ii) the second class constraints $\bar{\Phi}_{(2) \mathcal{A}_{2}} \approx 0, \mathcal{A}_{2}=1, . ., r_{2}$ $\left(r_{1}+r_{2}=M\right)$ with $\left\{\bar{\Phi}_{(2) \mathcal{A}_{2}}, \bar{\zeta}_{\mathcal{A}}\right\} \neq 0$ for some $\mathcal{A}$. Evidently we have $\left\{\bar{\Phi}_{(1) \mathcal{A}_{1}}, \bar{\Phi}_{(1) \mathcal{B}_{1}}\right\} \approx 0$, $\left\{\bar{\Phi}_{(1) \mathcal{A}_{1}}, \bar{\Phi}_{(2) \mathcal{A}_{2}}\right\} \approx 0, \operatorname{det}\left(\left\{\bar{\Phi}_{(2) \mathcal{A}_{2}}, \bar{\Phi}_{(2) \mathcal{B}_{2}}\right\}\right) \neq 0$.

The $\bar{\phi}_{\bar{A}}$ 's constitute a complete set of first class primary constraints.

If a set of first class constraints has the form $\bar{\Phi}_{(1) a}=p_{a}-\bar{K}_{a}\left(q^{a}, q^{r}, p_{r}\right) \approx 0$ with $r \neq a$ (i.e. they are solved in a subset of the momenta), then $\left\{\bar{\Phi}_{(1) a}, \bar{\Phi}_{(1) b}\right\}=\frac{\partial \bar{K}_{a}}{\partial q^{b}}-\frac{\partial \bar{K}_{b}}{\partial q^{a}}+\left\{\bar{K}_{a}, \bar{K}_{b}\right\} \equiv 0$. 
Geometrically the Hamiltonian vector fields $\bar{X}_{(1) \mathcal{A}_{1}}=\left\{., \bar{\Phi}_{(1) \mathcal{A}_{1}}\right\}$ and $\bar{X}_{(2) \mathcal{A}_{2}}=\left\{., \bar{\Phi}_{(2) \mathcal{A}_{2}}\right\}$ are tangent and skew respectively to the constraint sub-manifold $\bar{\gamma}$ (see Ref.[6]; in Ref.[26] there is a study of the conditions for putting all the first-class constraints in this Abelianized form as also discussed in Subsection IVC).

\section{Chains of Constraints: Diagonalization of the Dirac Algorithm.}

We quote three theorems $[14,27,28]$ on equivalent sets of constraints $\bar{\zeta}_{\mathcal{A}}(q, p) \approx 0$, $\tilde{\bar{\zeta}}_{\mathcal{A}}(q, p) \approx 0$ both defining $\bar{\gamma}$, valid when suitable regularity conditions hold, without reproducing the long not illuminating demonstrations based on inductive procedures. These theorems allow to perform a diagonalization of the Dirac algorithm and to separate the constraints in chains (one for each primary constraint, namely for each null eigenvalue of the Hessian matrix), such that the time constancy of a constraint in the chain implies the next constraint in the chain. The time constancy of the last constraint in the chain either is automatically satisfied or determines the Dirac multiplier associated to the chain. A 0-chain has only the primary constraint, a 1-chain has the primary and a secondary, and so on.

The first theorem shows the existence of diagonalized chains.

Theorem 1 [14] - By taking suitable combinations $\bar{\phi}_{\bar{A}}=\bar{V}^{(f-1)_{\bar{A}}^{A_{f-1}}} \ldots \bar{V}_{A_{1}}^{A} \bar{\phi}_{A}(\bar{A}=1, . ., \bar{k}=$ $\left.m-h_{1}-. .-h_{f}\right)$ and $\bar{\phi}_{A^{\prime}}=\bar{u}_{A^{\prime}}^{A} \bar{\phi}_{A}\left(A^{\prime}=1, . ., m-\bar{k}\right)$ of the primary constraints $\bar{\phi}_{A}$ $(A=1, . ., m)$ defining $\gamma$, then suitable combinations of the secondary $\bar{\chi}_{a_{1}}^{(1)}$ and primary $\bar{\phi}_{A}$ constraints defining $\gamma_{1}$ and so on, the final pattern of the chains of constraints can be put in the following form:

i) chains of constraints starting from primary constraints whose Dirac multiplier $\lambda^{A}(t)$ is determined by the Dirac algorithm on $\bar{\gamma}$. We use the following notation: $\bar{\phi}_{(h) A_{h}^{\prime}} \approx 0$ is the primary constraint of a $h$-chain of $h+1$ constraints ( $A_{h}^{\prime}$ labels the various $h$-chains), $\bar{\phi}_{(h) A_{h}^{\prime}}^{(1)} \approx$ $\frac{d \bar{\phi}_{(h) A_{h}^{\prime}}}{d t} \approx\left\{\bar{\phi}_{(h) A_{h}^{\prime}}, \bar{H}_{c}^{(1)}\right\} \approx 0$ is the secondary, $\bar{\phi}_{(h) A_{h}^{\prime}}^{(2)} \approx \frac{d \bar{\phi}_{(h) A_{h}^{\prime}}^{(1)}}{d t} \approx\left\{\bar{\phi}_{(h) A_{h}^{\prime}}^{(1)}, \bar{H}_{c}^{(2)}\right\} \approx 0$ is the tertiary and so on till $\bar{\phi}_{(h) A_{h}^{\prime}}^{\left(f_{h}\right)} \approx \frac{d \bar{\phi}_{(h) A_{h}^{\prime}}^{\left(f_{h}-1\right)}}{d t} \approx\left\{\bar{\phi}_{(h) A_{h}^{\prime}}^{\left(f_{h}-1\right)}, \bar{H}_{c}^{\left(f_{h}\right)}\right\} \approx 0$; then $\frac{d \bar{\phi}_{(h) A_{h}^{\prime}}^{\left(f_{h}\right)}}{d t} \approx 0$ determines the Dirac multiplier. Here $\bar{H}_{D}, \bar{H}_{c}, \bar{H}_{c}^{(1)}, .$. are the quantities already introduced in the previous Section.

$$
\begin{array}{llll}
0-\text { chains } & 1-\text { chains } & & f-\text { chains } \\
\bar{\phi}_{(o) A_{o}^{\prime}}\left(A_{o}^{\prime}=1, . ., k_{o}^{\prime}\right) & \bar{\phi}_{(1) A_{1}^{\prime}}\left(A_{1}^{\prime}=1, . ., k_{1}^{\prime}\right) & \ldots & \bar{\phi}_{(f) A_{f}^{\prime}}\left(A_{f}^{\prime}=1, . ., k_{f}^{\prime}\right) \\
\lambda^{(o) A_{o}^{\prime}} \text { determined } & \bar{\phi}_{(1) A_{1}^{\prime}}^{(1)} & \ldots & \bar{\phi}_{(f) A_{f}^{\prime}}^{(1)} \\
& \lambda^{(1) A_{1}^{\prime}} \text { determined } & \ldots & \bar{\phi}_{(f) A_{f}^{\prime}}^{(2)} \\
\ldots & \ldots & \ldots & \text { primary } \\
& \ldots & \ldots & \bar{\phi}_{(f) A_{f}^{\prime}}^{(f)} \\
& & \ldots & \lambda^{(f) A_{f}^{\prime}} \text { determined }
\end{array}
$$

ii) chains of constraints starting from the primary constraints $\bar{\phi}_{\bar{A}}$ with associated arbitrary Dirac multipliers on $\bar{\gamma}$. The same notation as in i) is used; but now $\frac{d \bar{\phi}_{(h)}^{\left(f_{h}\right)}}{d t} \approx 0$ is identically 
satisfied without determining the Dirac multiplier.

$$
\begin{aligned}
& \begin{array}{lll}
0-\text { chains } & 1-\text { chains } & f-\text { chains } \\
\bar{\phi}_{(o) \bar{A}_{o}}\left(\bar{A}_{o}=1, . ., k_{o}^{\prime}\right) & \bar{\phi}_{(1) \bar{A}_{1}}\left(\bar{A}_{1}=1, . ., k_{1}^{\prime}\right) & \bar{\phi}_{(f) \bar{A}_{f}}\left(\bar{A}_{f}=1, . ., k_{f}^{\prime}\right)
\end{array} \mid \text { primary } \\
& \begin{array}{lll}
\bar{\phi}_{(1) \bar{A}_{1}}^{(1)} \quad \ldots \bar{\phi}_{(f) \bar{A}_{f}}^{(1)} & \text { secondary }
\end{array} \\
& \ldots \bar{\phi}_{(f) \bar{A}_{f}}^{(2)} \quad \text { tertiary } \\
& \begin{array}{ll|l}
\cdots & \bar{\phi}_{(f)}^{(f)} \bar{A}_{f} & (f+1)-\operatorname{ary}
\end{array}
\end{aligned}
$$

The second theorem shows that the diagonalized chains of Theorem 1 can be redefined so that each chain has all the constraints either first or second class.

Theorem 2 [27] - By leaving the primary constraints in the form of Theorem 1, we can take linear combinations of all the other constraints so to obtain the following pattern:

i) chains of second class constraints $\bar{\chi}_{(k) A_{k}^{\prime}}^{(h)}$ with the associated Dirac multiplier determined $\left(\operatorname{det}\left(\left\{\bar{\chi}_{(k) A_{k}^{\prime}}^{(h)}, \bar{\chi}_{\left(k_{1}\right) A_{k_{1}}^{\prime}}^{\left(h_{1}\right)}\right\}\right) \neq 0\right)$.

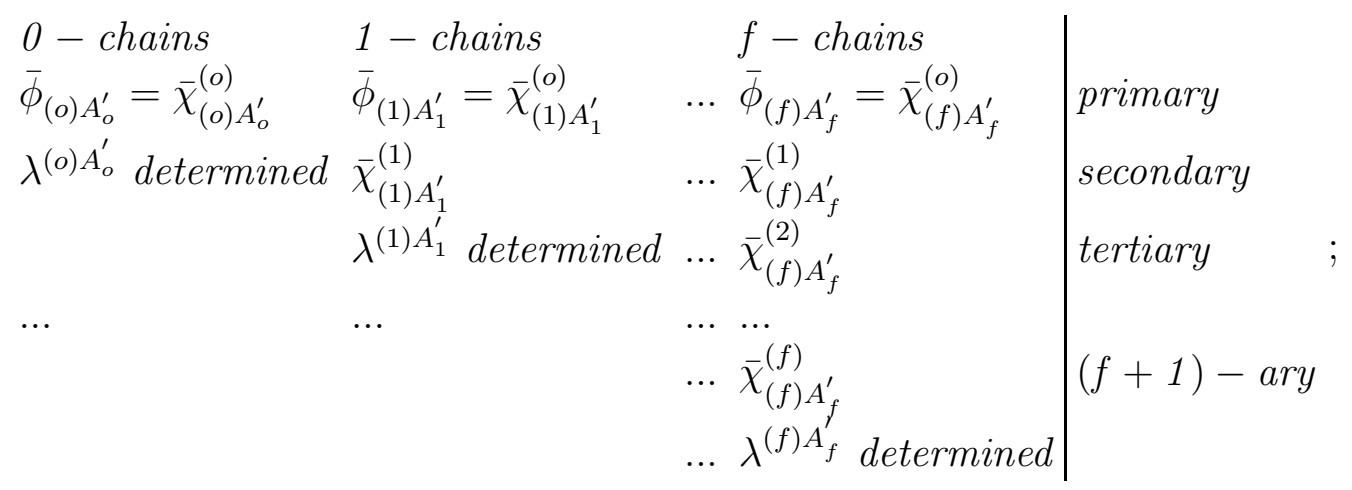

ii) chains of first class constraints $\bar{\chi}_{(k) \bar{A}_{k}}^{(h)}$ with arbitrary Dirac multipliers and with the property $\bar{\chi}_{(k) \bar{A}_{k}}^{(h+1)}=\left\{\bar{\chi}_{(k) \bar{A}_{k}}^{(h)}, \bar{H}_{c}^{(F)}\right\}$.

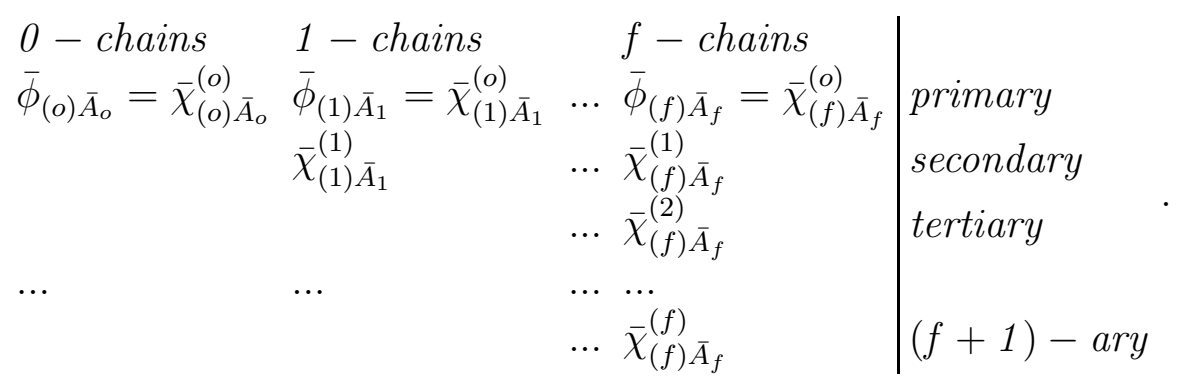

The third theorem gives a simple canonical form for the chains of second class constraints. Theorem $3[28]$ - By adding suitable terms quadratic in the second class constraints to $\bar{H}_{c}^{(F)}$ (this is irrelevant on $\bar{\gamma}$ ) to get a new $\bar{H}_{c}^{(F)^{\prime}}$ and by making an appropriate linear orthogonal transformation on the set of primary second class constraints $\bar{\phi}_{(k) A_{k}^{\prime}}=\bar{\chi}_{(k) A_{k}^{\prime}}^{(o)}$ to obtain the new primary second class constraints $\bar{\chi}_{(k) A_{k}^{\prime}}^{(o)^{\prime}}$, we get new forms $\bar{\chi}_{(k) A_{k}^{\prime}}^{(h)^{\prime}}$ of the non-primary 
second class constraints such that all the second class chains have the following canonical form, in which each constraint ${ }_{(a)} \bar{\chi}_{(k) A_{k}^{\prime}}^{(h)^{\prime}}$ has zero Poisson bracket with all the constraints except with a partner either in its chain or in another one with the same prefix $(a)$.

$$
\begin{aligned}
& \text { pairs of } 0-\text { chains } \quad{ }_{(1)} \bar{\chi}_{(o) A^{\prime}}^{(o)^{\prime}}{ }^{(1)} \bar{\chi}_{(o) B^{\prime} \neq A^{\prime}}^{(o)^{\prime}} \quad \text { primary } \\
& 1 \text {-chains } \quad \text { (1) } \bar{\chi}_{(1) A^{\prime}}^{(o)^{\prime}} \quad \text { primary } \\
& \text { (1) } \bar{\chi}_{(1) A^{\prime}}^{(1)^{\prime}} \quad \text { secondary } \\
& \text { pairs of } 2 \text { - chains }{ }_{(1)} \bar{\chi}_{(2) A^{\prime} \quad(2)}^{(o)^{\prime}} \bar{\chi}_{(2) B^{\prime} \neq A^{\prime}}^{(o)^{\prime}} \quad \text { primary } \\
& \text { (3) } \bar{\chi}_{(2) A^{\prime}(3)}^{(1)^{\prime}} \bar{\chi}_{(2) B^{\prime} \neq A^{\prime}}^{(1)^{\prime}} \text { secondary } \\
& { }_{(2)} \bar{\chi}_{(2) A^{\prime}}^{(2)} \quad(1) \bar{\chi}_{(2) B^{\prime} \neq A^{\prime}}^{(2)^{\prime}} \text { tertiary } \\
& 3 \text { - chains } \quad \text { (1) } \bar{\chi}_{(3) A^{\prime}}^{(o)^{\prime}} \quad \text { primary } \\
& \text { (2) } \bar{\chi}_{(3) A^{\prime}}^{(1)^{\prime}} \quad \text { secondary } \\
& \text { (2) } \bar{\chi}_{(3) A^{\prime}}^{(2)^{\prime}} \quad \text { tertiary } \\
& \text { (1) } \bar{\chi}_{(3) A^{\prime}}^{(3)^{\prime}} \quad \text { quaternary } \\
& \text { pairs of } \left.4 \text { - chains }{ }_{(1)} \bar{\chi}_{(4) A^{\prime}}^{(o)^{\prime}} \quad{ }^{2}\right) \bar{\chi}_{(4) B^{\prime} \neq A^{\prime}}^{(o)^{\prime}} \text { primary } \\
& \text { (3) } \bar{\chi}_{(4) A^{\prime}(4)}^{(1)^{\prime}} \bar{\chi}_{(4) B^{\prime} \neq A^{\prime}}^{(1)^{\prime}} \text { secondary } \\
& { }_{(5)} \bar{\chi}_{(4) A^{\prime} \quad(5)}^{(2)^{\prime}} \bar{\chi}_{(4) B^{\prime} \neq A^{\prime}}^{(2)^{\prime}} \text { tertiary } \\
& { }_{(4)} \bar{\chi}_{(4) A^{\prime} \quad(3)}^{(3)^{\prime}} \bar{\chi}_{(4) B^{\prime} \neq A^{\prime}}^{(3)^{\prime}} \quad \text { quaternary } \\
& { }_{(2)} \bar{\chi}_{(4) A^{\prime} \quad(1)}^{(4)^{\prime}} \bar{\chi}_{(4) B^{\prime} \neq A^{\prime}}^{(4)^{\prime}} \quad 5-\text { ary } \\
& 5 \text { - chains } \quad(1) \bar{\chi}_{(5) A^{\prime}}^{(o)^{\prime}} \quad \text { primary } \\
& \text { (2) } \bar{\chi}_{(5) A^{\prime}}^{(1)^{\prime}} \quad \text { secondary } \\
& \text { (3) } \bar{\chi}_{(5) A^{\prime}}^{(2)^{\prime}} \quad \text { tertiary } \\
& { }_{(3)} \bar{\chi}_{(5) A^{\prime}}^{(3)^{\prime}} \quad \text { quaternary } \\
& \text { (2) } \bar{\chi}_{(5) A^{\prime}}^{(4)^{\prime}} \quad 5-\text { ary } \\
& { }_{(1)} \bar{\chi}_{(5) A^{\prime}}^{(5)^{\prime}} \quad 6-\text { ary, }
\end{aligned}
$$

and so on with this alternating pattern from even-chains to odd-chains.

This theorem shows that under suitable regularity conditions on the singular Lagrangian we cannot obtain neither a chain in which a primary first class constraints generates a secondary second class constraint nor two chains, whose primary constraints are a second class pair and which generate secondary first class constraints. 


\section{E. Second Class Constraints and Dirac Brackets.}

Second class constraints describe inessential pairs of canonical variables, which can be eliminated reducing the number of the degrees of freedom carrying the dynamics of the singular system (maybe with the price of a breaking of manifest covariance and/or of the introduction of non-linearities).

When we have a singular system with the constraint sub-manifold $\bar{\gamma} \subset T^{*} Q$ described by the set $\tilde{\bar{\zeta}}_{\mathcal{A}}=\left(\bar{\Phi}_{(1) \mathcal{A}_{1}}, \bar{\Phi}_{(2) \mathcal{A}_{2}}\right)$ of first $\left(\bar{\Phi}_{(1) \mathcal{A}_{1}}\right), \mathcal{A}_{1}=1, . ., m-2 s_{2}$, and second $\left(\bar{\Phi}_{(2) \mathcal{A}_{2}}\right)$, $\mathcal{A}_{2}=1, . ., 2 s_{2}$, class constraints, we can (at least implicitly) eliminate $s_{2}$ pairs of canonical variables with the following procedure. Let us assume that the second class constraints define a $2\left(n-s_{2}\right)$-dimensional constraint sub-manifold $\gamma_{(2)}$ of $T^{*} Q$ containing the final submanifold $\bar{\gamma} \subset \gamma_{(2)} \subset T^{*} Q$ and that the regularity conditions are such that the anti-symmetric matrix

$$
\tilde{\bar{C}}_{\mathcal{A}_{2} \mathcal{B}_{2}}=\left(\left\{\bar{\Phi}_{(2) \mathcal{A}_{2}}, \bar{\Phi}_{(2) \mathcal{B}_{2}}\right\}\right)
$$

which is invertible on $\bar{\gamma}\left(\left.\operatorname{det} \tilde{\bar{C}}_{\mathcal{A}_{2} \mathcal{B}_{2}}\right|_{\bar{\gamma}} \neq 0\right)$, is invertible also on $\gamma_{(2)}\left(\left.\operatorname{det} \tilde{\bar{C}}_{\mathcal{A}_{2} \mathcal{B}_{2}}\right|_{\gamma_{(2)}} \neq 0\right)$ with inverse matrix $\tilde{\bar{C}}^{\mathcal{A}_{2} \mathcal{B}_{2}}$ on $\gamma_{(2)}$. As shown by Dirac [1], the sub-manifold $\gamma_{(2)}$ (in general it is not $T^{*} Q_{(2)}$ for some configuration space $\left.Q_{(2)}\right)$ has an induced symplectic structure whose Poisson brackets, named Dirac brackets, are

$$
\{\bar{f}, \bar{g}\}^{*}=\{\bar{f}, \bar{g}\}-\left\{\bar{f}, \bar{\Phi}_{(2) \mathcal{A}_{2}}\right\} \tilde{\bar{C}}^{\mathcal{A}_{2} \mathcal{B}_{2}}\left\{\bar{\Phi}_{(2) \mathcal{A}_{2}}, \bar{g}\right\} .
$$

Besides the standard properties $\{\bar{f}, \bar{g}\}^{*}=-\{\bar{g}, \bar{f}\}^{*},\left\{\bar{f}, \bar{g}_{1} \bar{g}_{2}\right\}^{*}=\left\{\bar{f}, \bar{g}_{1}\right\}^{*} \bar{g}_{2}+\bar{g}_{1}\left\{\bar{f}, \bar{g}_{2}\right\}^{*}$, $\left\{\{\bar{f}, \bar{g}\}^{*}, \bar{u}\right\}^{*}+\left\{\{\bar{u}, \bar{f}\}^{*}, \bar{g}\right\}^{*}+\left\{\{\bar{g}, \bar{u}\}^{*}, \bar{f}\right\}^{*}=0$, the Dirac brackets have the extra, easily verified, properties

$$
\begin{aligned}
& \left\{\bar{\Phi}_{(2) \mathcal{A}_{2}}, \bar{f}\right\}^{*}=0 \quad \text { for every } \bar{f} \text { and } \mathcal{A}_{2}, \Rightarrow \quad \bar{\Phi}_{(2) \mathcal{A}_{2}} \equiv 0 \quad \text { on } \gamma_{(2)}, \\
& \left\{\bar{f}, \bar{g}_{(1)}\right\}^{*} \approx\left\{\bar{f}, \bar{g}_{(1)}\right\} \quad \text { on } \bar{\gamma} \subset \gamma_{(2)}, \text { for } \bar{g}_{(1)} \text { first class, } \bar{f} \text { arbitrary, } \\
& \left\{\bar{f},\left\{\bar{g}_{(1)}, \bar{u}_{(1)}\right\}^{*}\right\}^{*} \approx\left\{\bar{f},\left\{\bar{g}_{(1)}, \bar{u}_{(1)}\right\}\right\} \quad \text { on } \bar{\gamma} \subset \gamma_{(2)}, \\
& \text { for } \bar{g}_{(1)} \text { and } \bar{u}_{(1)} \text { first class, } \bar{f} \text { arbitrary. }
\end{aligned}
$$

When we use the Dirac brackets, the Dirac Hamiltonian $\bar{H}_{D}=\bar{H}_{c}^{(F)}+\lambda^{\bar{A}}(t) \bar{\phi}_{\bar{A}}$ becomes $\bar{H}_{D}^{\prime}=\bar{H}_{c}^{(F)^{\prime}}+\lambda^{\bar{A}}(t) \bar{\phi}_{\bar{A}}$ with $\bar{H}_{c}^{(F)^{\prime}}=\left.\bar{H}_{c}^{(F)}\right|_{\gamma_{(2)}}$. Since the Dirac Hamiltonian is a first class function, for the Hamilton-Dirac equations we get $\frac{d \bar{f}}{d t} \stackrel{\circ}{=}\left\{\bar{f}, \bar{H}_{D}\right\} \approx\left\{\bar{f}, \bar{H}_{D}^{\prime}\right\}^{*}$ on $\bar{\gamma} \subset \gamma_{(2)}$.

The second class constraints are not generators of canonical transformations interpretable as Hamiltonian gauge transformations like first class constraints (see next Section), but, as we shall see in Section VI, they are the generators of local Noether extended symmetry transformations under which the singular Lagrangian has a generalized type of quasi-invariance (instead the first class constraints generate local Noether symmetry transformations under which the singular Lagrangian is quasi-invariant). 


\section{HAMILTONIAN GAUGE TRANSFORMATIONS AND DIRAC OBSEERV- ABLES}

In this Section we analyze the Hamiltonian gauge transformations generated by the firstclass constraints, the notion of DO and the canonical transformations for the identification of a canonical Darboux basis adapted to both first- and second-class constraints.

\section{A. First Class Constraints and Hamiltonian Gauge Transformations.}

On the final constraint sub-manifold $\bar{\gamma} \subset T^{*} Q$ the Dirac Hamiltonian depends on as many arbitrary Dirac multipliers $\lambda^{\bar{A}}(t)$ as primary first class constraints $\bar{\phi}_{\bar{A}}(q, p) \approx 0$. As a consequence the solutions $q^{i}(t), p_{i}(t)$ of the Hamilton-Dirac equations are functionals of these arbitrary functions of time and cannot correspond to measurable observables, which must have a deterministic dependence on time starting from a given set of Cauchy initial data.

Let us give the canonical coordinates $q_{o}^{i}=q^{i}\left(t_{o}\right), p_{o i}=p_{i}\left(t_{o}\right)$ at time $t_{o}$ : this is interpreted as giving a physical initial state for the system. Let us consider the time evolution of a function $\bar{f}(q, p)$ from $t_{o}$ to $t_{o}+\delta t$ generated by the Dirac Hamiltonian: $\left.\left.\bar{f}(q, p)\right|_{t_{o}+\delta t} \stackrel{\circ}{=} \bar{f}(q, p)\right|_{t_{o}}+$ $\delta t\left\{\bar{f}(q, p), \bar{H}_{D}(q, p, \lambda)\right\}=\left.\bar{f}(q, p)\right|_{t_{o}}+\delta t\left\{\bar{f}(q, p), \bar{H}_{c}^{(F)}(q, p)\right\}+\delta t \lambda^{\bar{A}}(t)\left\{\bar{f}(q, p), \bar{\phi}_{\bar{A}}(q, p)\right\}$. If we consider two sets of Dirac multipliers $\lambda_{1}^{\bar{A}}(t)$ and $\lambda_{2}^{\vec{A}}(t)$ coinciding at $t_{o}\left[\lambda_{1}^{\bar{A}}\left(t_{o}\right)=\lambda_{2}^{\vec{A}}\left(t_{o}\right)\right]$, we obtain the result that at time $t_{o}+\delta t$ there is no uniquely determined value for $q^{i}\left(t_{o}+\delta t\right)$, $p_{i}\left(t_{o}+\delta t\right)$, because for every function we get the following difference between the two time evolutions $\left.\triangle_{12} \bar{f}(q, p)\right|_{t_{o}+\delta t}=\left.\delta t\left[\lambda_{1}^{\bar{A}}\left(t_{o}\right)-\lambda_{2}^{\bar{A}}\left(t_{o}\right)\right]\left\{\bar{f}(q, p), \bar{\phi}_{\bar{A}}(q, p)\right\}\right|_{t_{o}}$.

The only way to recover a deterministic description of the physical states of the system like in the regular case is to abandon the assumption that a physical state is uniquely identified by one and only one set of values of the canonical coordinates at a given time. In the singular case at each instant of time many sets of canonical coordinates describe the same physical state. Two sets of canonical coordinates whose difference is $\triangle_{12} q^{i}, \triangle_{12} p_{i}$ are said to be gauge equivalent and the term $\lambda^{\bar{A}}(t) \bar{\phi}_{\bar{A}}(q, p)$ of the Dirac Hamiltonian is interpreted as the generator of a Hamiltonian gauge transformation. Therefore the $m$ primary first class constraints $\bar{\phi}_{\bar{A}}$ are the generators of the Hamiltonian gauge transformations responsible of the non-deterministic time evolution. This means that in the singular case we must:

i) Find which is the maximal set of Hamiltonian gauge transformations existing for each given singular system besides those appearing in the Dirac Hamiltonian (they are the only ones allowed in the description of the time evolution).

ii) Separate the canonical variables in three disjoint sets:

a) the Hamiltonian gauge invariant variables (the so called Dirac observables, DO), which have deterministic time evolution so that a complete set of them at one instant identifies the physical state of the system at that instant;

b) the inessential pairs of canonical variables eliminable by means of the second class constraints with the Dirac brackets;

c) the Hamiltonian gauge variables which are irrelevant for the identification of a physical state, because they have an arbitrary time evolution. The number of gauge variables will coincide with the number of functionally independent generators of infinitesimal Hamiltonian gauge transformations, which allow the reconstruction of their maximal set.

To find the maximal set of infinitesimal Hamiltonian gauge transformations we shall assume that their generators $\bar{G}_{a}(q, p), a=1, . ., g$ have the structure of a local Hamiltonian 
gauge algebra $\tilde{g}$ under the Poisson brackets, namely $\left\{\bar{G}_{a}(q, p), \bar{G}_{b}(q, p)\right\}=\bar{C}_{a b}{ }^{c}(q, p) \bar{G}_{c}(q, p)$ with some set of structure functions $\bar{C}_{a b}{ }^{c}(q, p)$. When the structure functions are constant on $\bar{\gamma}, \bar{C}_{a b}{ }^{c}=C_{a b}{ }^{c}=$ const., we speak of a Lie gauge algebra with structure constants $C_{a b}{ }^{c}$.

Once the generators $\bar{G}_{a}$ 's are known the next problem is to define a local Hamiltonian gauge group $\mathcal{G}$, i.e. the finite Hamiltonian gauge transformations which can be build with sequences of infinitesimal Hamiltonian gauge transformations, and then to see whether there exist Hamiltonian gauge transformations not connected to the identity (large gauge transformations) due to the topological properties of the system. The Hamiltonian gauge group is said to be local, because the space of its gauge parameters (i.e. the coordinates of its group manifold) is coordinatized by arbitrary functions of time $\epsilon^{a}(t), a=1, . ., g$, and not by numerical constants $\epsilon^{a}=$ const.

Let us remark that the Hamiltonian gauge transformations are defined off-shell, namely without using the equations of motion, and that in general they are more general of the standard Lagrangian gauge transformations. Only on-shell, namely on the space of the solutions of the equations of motion, do the two notions of gauge transformations coincide and both of them are then also gauge dynamical symmetries of the equations of motion.

The primary first class constraints $\bar{\phi}_{\bar{A}}(q, p)$ are in general only a subset of the $\bar{G}_{a}$ 's. Their associated gauge parameters $\epsilon^{a=\bar{A}}(t)$ are the Dirac multipliers $\lambda^{\bar{A}}(t) \stackrel{\circ}{=} g_{(\lambda)}^{\bar{A}}(q, \dot{q})$, which identify the non-determined non-projectable velocity functions of the singular system associated with the non-invertibility of the equations $p_{i}=\mathcal{P}_{i}(q, \dot{q})$. Therefore there will be an equal number of primary Hamiltonian gauge variables $\bar{Q}^{\bar{A}}(q, p)$, which are transformed among themselves by the Hamiltonian gauge transformations generated by the $\bar{\phi}_{\bar{A}}$ 's, satisfy $\frac{d \bar{Q}^{\bar{A}}}{d t} \stackrel{\circ}{=}\left\{\bar{Q}^{\bar{A}}, \bar{H}_{D}\right\}=$ $\lambda^{\bar{A}}(t) \stackrel{\circ}{=} g_{(\lambda)}^{\bar{A}}(q, \dot{q})$ and do not contribute to the identification of the physical state of the system.

However, the study of the Hamilton-Dirac equations (or of the EL equations) will show that in general there exist secondary Hamiltonian (or Lagrangian) gauge variables $\overline{\mathcal{T}}^{\alpha}(q, p)$, which inherit the arbitrariness of the Dirac multipliers, being functionals of them on the solutions of the equations of motion. This implies that the gauge parameters $\epsilon^{a}(t), a=$ $1, \ldots, g$, of the off-shell Hamiltonian gauge transformations have to be restricted to $\epsilon^{a=\bar{A}}(t)=$ $\lambda^{\bar{A}}(t), \epsilon^{a \neq \bar{A}}(t)=F^{a \neq \bar{A}}\left[\lambda^{\bar{B}}(t)\right]$ to be interpretable as the gauge parameters of the on-shell Hamiltonian gauge group.

The gauge algebra assumption implies that the Poisson bracket of two infinitesimal gauge transformations must be a gauge transformation. Therefore we must have $\left\{\bar{\phi}_{\bar{A}}(q, p), \bar{\phi}_{\bar{B}}(q, p)\right\}=\bar{C}_{\bar{A} \bar{B}}{ }^{a}(q, p) \bar{G}_{a}(q, p)$.

Moreover the gauge algebra must not change in time. This implies that the time derivative $\frac{d \bar{G}_{a}}{d t} \stackrel{\circ}{=}\left\{\bar{G}_{a}, \bar{H}_{D}\right\}$ of a Hamiltonian gauge transformation must be again a gauge transformation. Since we only know that $\left\{\bar{\phi}_{\bar{A}}, \bar{\phi}_{\bar{B}}\right\}=\bar{C}_{\bar{A} \bar{B}}{ }^{a} \bar{G}_{a}$, we get the following requirement on the $\bar{\phi}_{\bar{A}}$ 's: $\left\{\bar{\phi}_{\bar{A}}(q, p), \bar{H}_{c}^{(F)}(q, p)\right\}=\bar{V}_{\bar{A}}^{a}(q, p) \bar{G}_{a}(q, p)$, where $\bar{H}_{c}^{(F)}$ is the final canonical Hamiltonian (a first class quantity).

Let us assume that the regularity conditions on the singular Lagrangian be such that Theorem 2 of Subsection IIID on the diagonalization of chains of constraints holds. This means that we can find linear first class combinations $\bar{\chi}_{(k)}^{(o)} \bar{A}_{k}$ of the primary constraints such that $\left\{\bar{\chi}_{(k) \bar{A}_{k}}^{(o)}, \bar{H}_{c}^{(F)}\right\}=\bar{\chi}_{(k) \bar{A}_{k}}^{(1)}$. Therefore in this form the secondary first class constraints are generators of Hamiltonian gauge transformations. The general result $\left\{\bar{\chi}_{(k) \bar{A}_{k}}^{(h)}, \bar{H}_{c}^{(F)}\right\}=$ $\bar{\chi}_{(k) \bar{A}_{k}}^{(h+1)}$ shows that all the secondary, tertiary ... first class constraints are generators of Hamiltonian gauge transformations, namely that $\bar{G}_{\mathcal{A}}=\bar{\Phi}_{(1) \mathcal{A}}, \mathcal{A}=1, . ., M$. Since in general 
$\left\{\bar{\Phi}_{(1) \mathcal{A}}, \bar{\Phi}_{(1) \mathcal{B}}\right\}=\bar{C}_{\mathcal{A B}}{ }^{\mathcal{C}} \bar{\Phi}_{(1) \mathcal{C}}+\bar{C}_{\mathcal{A B}}^{\prime}{ }^{\mathcal{C}^{\prime}} \bar{\Phi}_{(2) \mathcal{C}^{\prime}}$, we see that a true gauge algebra is obtained only near the second class sub-manifold $\gamma_{(2)}$, which contains the final constraint sub-manifold $\bar{\gamma}$.

Therefore under suitable regularity conditions on the singular Lagrangian Dirac's conjecture [1] that all the first class constraints are generators of Hamiltonian gauge transformations is true. Dirac also proposed to replace the final Dirac Hamiltonian $\bar{H}_{D}=$ $\bar{H}_{c}^{(F)}+\lambda^{\bar{A}}(t) \bar{\phi}_{\bar{A}}$ with the extended Hamiltonian

$$
\bar{H}_{E}=\bar{H}_{c}^{(F)}+\epsilon^{\mathcal{A}}(t) \bar{\Phi}_{(1) \mathcal{A}},
$$

including all the first class constraints each one with an arbitrary multiplier. In this way the time evolution is split in a deterministic part governed by the final canonical Hamiltonian $\bar{H}_{c}^{(F)}$ (it generates a mapping from a gauge orbit to another one) and in a gauge part, which is the generator of the most general off-shell Hamiltonian gauge transformation.

Even if this extension does not change the on-shell dynamics (so that, as shown in Refs. $[6,8,9]$, it is taken as the starting point of the BRST quantization program), it has the drawback that its inverse Legendre transformation does not reproduce the original singular Lagrangian, because the secondary gauge variables have an arbitrary gauge freedom instead of the reduced one $\left(\epsilon^{\mathcal{A}=\bar{A}}(t)=\lambda^{\bar{A}}(t), \epsilon^{\mathcal{A} \neq \bar{A}}(t)=F^{\mathcal{A} \neq \bar{A}}\left[\lambda^{\bar{B}}(t)\right]\right)$ associated with the on-shell Hamiltonian gauge transformations. Even if they have a reduced gauge freedom, this is a consequence of the fact that the secondary gauge variables have non-vanishing Poisson brackets with the generators $\bar{G}_{\mathcal{A}}$. The results: i) $\left\{\bar{\chi}_{(k) \bar{A}_{k}}^{(h)}, \bar{H}_{c}^{(F)}\right\}=\bar{\chi}_{(k) \bar{A}_{k}}^{(h+1)}$ of Theorem 2; ii) $\bar{H}_{c}^{(F)}=\bar{H}_{c}+$ (combinations of primary both first and second class constraints), imply that all the secondary first class constraints $\bar{\chi}_{(k) \bar{A}_{k}}^{(h)}, h \neq 0$, must already be present in the original canonical Hamiltonian $\bar{H}_{c}$ with some form of the primary $\bar{Q}_{(k)}^{\bar{A}}=\overline{\mathcal{T}}_{(k)}^{(o) \bar{A}_{k}}$ and secondary $\overline{\mathcal{T}}_{(k)}^{(h) \bar{A}_{k}}, h>0$, gauge variables as coefficients (only the $\overline{\mathcal{T}}_{(k)}^{\left(f_{k}\right) \bar{A}_{k}}$ are not present in $\bar{H}_{c}$ )

$$
\bar{H}_{c}(q, p)=\bar{H}_{c}^{\prime}(q, p)+\sum_{k} \bar{Q}_{(k)}^{\bar{A}=\bar{A}_{k}}(q, p) \bar{\chi}_{(k) \bar{A}_{k}}^{(1)}(q, p)+\sum_{k} \sum_{h=1}^{f_{k}-1} \overline{\mathcal{T}}_{(k)}^{(h) \bar{A}_{k}}(q, p) \bar{\chi}_{(k) \bar{A}_{k}}^{(h+1)}(q, p) .
$$

For instance, this is what happens in field theories like electromagnetism, Yang-Mills theory and metric gravity, which have the secondary first class constraints already present in the canonical Hamiltonian density with in front the primary gauge variables.

Therefore Dirac's proposal (4.1) is already fulfilled for this class of singular Lagrangians, but with the gauge parameters of the on-shell Hamiltonian gauge group replacing those of the off-shell group present in the extended Hamiltonian.

In Ref.[25] it is shown that also the secondary, tertiary ... second class constraints of this class of singular Lagrangians are present in the canonical Hamiltonian $\bar{H}_{c}$ in the form of quadratic combinations. This is clear if we use the form $\bar{\chi}_{(k) A_{k}^{\prime}}^{(h)^{\prime}}$ of the second class constraints given in Theorem 3. Since these constraints are generated by the equations $\left\{\bar{\chi}_{(k) A_{k}^{\prime}}^{(h)^{\prime}}, \bar{H}_{D}^{(F)}\right\}=$ $\bar{\chi}_{(k) A_{k}^{\prime}}^{(h+1)^{\prime}}$, the final form of $\bar{H}_{c}$ implying these results will be

$$
\begin{aligned}
\bar{H}_{c}(q, p) & =\bar{H}_{d}(q, p)+\sum_{k}\left(\bar{Q}_{(k)}^{\bar{A}=\bar{A}_{k}}(q, p) \bar{\chi}_{(k) \bar{A}_{k}}^{(1)}(q, p)+\sum_{h=1}^{f_{k}-1} \overline{\mathcal{T}}_{(k)}^{(h) \bar{A}_{k}}(q, p) \bar{\chi}_{(k) \bar{A}_{k}}^{(h+1)}(q, p)\right)+ \\
& +\sum_{k, h_{1}, h_{2}} \bar{S}_{(k)}^{\left(h_{1} h_{2}\right) A_{k}^{\prime} B_{k}^{\prime}}(q, p) \bar{\chi}_{(k) A_{k}^{\prime}}^{\left(h_{1}\right)^{\prime}}(q, p) \bar{\chi}_{(k) B_{k}^{\prime}}^{\left(h_{2}\right)^{\prime}}(q, p) .
\end{aligned}
$$


with suitable functions $\bar{S}_{(k)}^{\left(h_{1} h_{2}\right) A_{k}^{\prime} B_{k}^{\prime}}$ consistent with the pattern of Poisson brackets of the second class constraints given in Theorem 3. In Eq.(4.3) $\bar{H}_{d}$ is the real first class deterministic Hamiltonian generating a mapping among the gauge orbits.

See the bibliography of Refs. $[25,29,30]$ for the attempts to prove Dirac's conjecture and the use of the extended Hamiltonian. However in many of these papers one uses singular Lagrangians with a singular Hessian matrix with non constant rank (see Section VI).

\section{B. Dirac Observables, Reduced Phase Space and Gauge Fixings.}

We have found that the generators $\bar{G}_{\mathcal{A}}$ of the maximal set of Hamiltonian gauge transformations are all the first class constraints $\bar{\Phi}_{(1) \mathcal{A}}, \mathcal{A}=1, . ., M$. Therefore, as already said, from the $2 n$ original canonical variables $q^{i}, p_{i}$ we can form three groups of functions:

i) $2 s_{2}$ functions which represent the inessential degrees of freedom eliminable by going to Dirac brackets with respect to the second class constraints $\bar{\Phi}_{(2) \mathcal{A}^{\prime}} \approx 0, \mathcal{A}^{\prime}=1, . ., 2 s_{2}$. They do not determine the physical state of the system, but only restrict the allowed region of $T^{*} Q$ to the second class $2\left(n-s_{2}\right)$-dimensional sub-manifold $\gamma_{(2)}$, on which the symplectic structure is given by the Dirac brackets, by eliminating degrees of freedom with trivial first order dynamics. The Dirac Hamiltonian on $\gamma_{(2)}$ is $\left.\bar{H}_{D}\right|_{\gamma_{(2)}}=\bar{H}_{d}+$ (terms in the first class constraints). ii) The non-deterministic $M$ primary and secondary gauge variables, which also do not determine the physical state. Together with the first class constraints $\bar{\Phi}_{(1) \mathcal{A}} \approx 0, \mathcal{A}=$ $1, . ., M$, they form a set of $2 M$ functions not carrying dynamical information (except maybe a topological one). These constraints determine the final $\left[2\left(n-s_{2}\right)-M\right]$-dimensional submanifold $\bar{\gamma} \subset \gamma_{(2)} \subset T^{*} Q$. This sub-manifold, which can be odd-dimensional, does not admit a symplectic structure (no uniquely defined Poisson brackets exist for the functions on $\bar{\gamma}$ ) and is called a presymplectic (or co-isotropic) manifold co-isotropically embedded in $T^{*} Q$ $[12,13]$. When suitable mathematical requirements are satisfied, it can be shown that the sub-manifold $\bar{\gamma}$ is foliated by $M$-dimensional diffeomorphic leaves, the Hamiltonian gauge orbits.

iii) $2\left(n-M-s_{2}\right)$ independent functions $\bar{F}_{\alpha}(q, p)$ with (in general weakly) zero Poisson bracket with all the constraints, $\left\{\bar{F}_{\alpha}, \bar{\Phi}_{(1) \mathcal{A}}\right\} \approx 0,\left\{\bar{F}_{\alpha}, \bar{\Phi}_{(2) \mathcal{A}^{\prime}}\right\} \approx 0$. They are the gauge invariant classical Dirac observables (DO) which parametrize the physical states of the system. The DO's are those functions on $\bar{\gamma}$ which are constant on the gauge orbits. One DO is the deterministic part $\bar{H}_{d}$ of the canonical Hamitonian $\bar{H}_{c}$. If $\bar{F}, \bar{G}$ are DO's, then the Jacobi identity implies that also $\{\bar{F}, \bar{G}\}$ is a DO. Usually one eliminates the second class constraints by introducing the associated Dirac brackets $\{., .\}^{*}$ and considering $\bar{\gamma}$ a sub-manifold of the second class sub-manifold $\gamma_{(2)}$.

In the case that the constraint sub-manifold $\bar{\gamma}$ is foliated by $M$-dimensional diffeomorphic Hamiltonian gauge orbits (nice foliation), we can go to the quotient with respect to the foliation and define the reduced phase space $\bar{\gamma}_{R}$, which will be a manifold if the projection $\pi: \bar{\gamma} \mapsto \bar{\gamma}_{R}$ is a submersion, but in general not a co-tangent bundle $T^{*} Q_{R}$ over some reduced configuration space $Q_{R}$. In the nice case the reduced phase space is a symplectic manifold with a closed symplectic two-form and the Hamiltonian in $\bar{\gamma}_{R}$ is the deterministic part $\bar{H}_{d}$ of the canonical Hamiltonian and the Hamilton equations for the abstract DO's are $\frac{d \bar{F}_{R}}{d t} \stackrel{\circ}{=}\left\{\bar{F}_{R}, \bar{H}_{d R}\right\}_{R}=\left\{\bar{F}, \bar{H}_{d}\right\}^{*}$.

In general things may be much more complicated: there can be not diffeomorphic gauge orbits, there can be singular points in $\bar{\gamma}, \pi: \bar{\gamma} \mapsto \bar{\gamma}_{R}$ may not be a submersion, ... For more 
details see Ref.[6].

To avoid technical problems with the definition of the reduced phase space $\bar{\gamma}_{R}$, usually we try to build a copy of it by adding $M$ gauge fixing constraints $\bar{\rho}_{\mathcal{A}}(q, p) \approx 0, \mathcal{A}=1, . ., M$, to eliminate the gauge freedom by choosing a definite gauge. The constraints $\bar{\rho}_{\mathcal{A}}(q, p) \approx 0$, $\bar{\Phi}_{(1) \mathcal{A}}(q, p) \approx 0$ must form a second class set and we can define their Dirac brackets. Locally the hyper-surface $\bar{\rho}_{\mathcal{A}}(q, p) \approx 0$ in $T^{*} Q$ should intersect each Hamiltonian gauge orbit in $\bar{\gamma}$ in one and only one point (modulo global problems like the Gribov ambiguity in Yang-Mills theory [6]).

If we use the Dirac Hamiltonian (namely the on-shell gauge group), we have only $\bar{k} \leq m$ Dirac multipliers $\lambda^{\bar{A}}(t)$ in front of the primary first class constraints $\bar{\phi}_{\bar{A}}(q, p) \approx 0$. The procedure for introducing the gauge fixing constraints in this case has been delineated in Ref.[31]. Let us use the notation $\bar{\Phi}_{(1) \mathcal{A}}=\left(\bar{\chi}_{(k) \bar{A}_{k}}^{(h)}\right), h=1, . ., f_{k}$, of Theorem 2 for the constraints. Each $f_{k}$-chain of first class constraints starts with the primary $\bar{\chi}_{(k) \bar{A}_{k}}^{(o)}$ [its associated Dirac multiplier is denoted $\left.\lambda_{(k)}^{\bar{A}_{k}}(t)\right]$ and ends with the $\left(f_{k}+1\right)$-ary constraint $\bar{\chi}_{(k) \bar{A}_{k}}^{\left(f_{k}\right)}$. Let us add as many gauge fixing constraints $\bar{\rho}_{(k) \bar{A}_{k}}^{\left(f_{k}\right)} \approx 0$ as $\left(f_{k}+1\right)$-ary constraints. It must be $\operatorname{det}\left(\left\{\bar{\rho}_{(k) \bar{A}_{k}}^{\left(f_{k}\right)}, \bar{\chi}_{(h) \bar{A}_{h}}^{\left(f_{h}\right)}\right\}\right) \neq 0$ and, for the sake of simplicity, we assume that $\left\{\bar{\rho}_{(k) \bar{A}_{k}}^{\left(f_{k}\right)}, \bar{\chi}_{(h) \bar{A}_{h}}^{(o)}\right\}=0$ for all $h, \bar{A}_{h}$. The requirement that the gauge fixings are preserved in time, i.e. $\frac{d \bar{\rho}_{(k) \bar{A}_{k}}^{\left(f_{k}\right)}}{d t} \stackrel{\circ}{=}\left\{\bar{\rho}_{(k) \bar{A}_{k}}^{\left(f_{k}\right)}, \bar{H}_{D}\right\}=\bar{\rho}_{(k) \bar{A}_{k}}^{\left(f_{k}-1\right)} \approx 0$, generates the gauge fixing constraints $\bar{\rho}_{(k) \bar{A}_{k}}^{\left(f_{k}-1\right)} \approx 0$ to the $f_{k}$-ary constraints $\bar{\chi}_{(k) \bar{A}_{k}}^{\left(f_{k}-1\right)} \approx 0$. The preservation in time of these induced gauge fixings generates the gauge fixing constraints for the $\left(f_{k}-1\right)$-ary first class constraints and so on. Each time we arrive at the gauge fixing of a primary first class constraint, its preservation in time determines the Dirac multiplier associated with the chain. In other words, we first fix the value of the $\left(f_{k}+1\right)$-ary gauge variables, its time constancy fixes the $f_{k}$-ary gauge variables and so on; the preservation in time of the gauge fixing of the primary gauge variables determines the Dirac multipliers.

Let us remark that due to the difficulties in trying to quantize the Dirac brackets after the elimination of an arbitrary set of second class constraints, there have been some attempts to redefine the theory in such a way that only first class constraints are present. The gauge fixings to the gauge freedom associated with these new constraints reproduce the original theory with its second class constraints. The method [32-34] (see also exercise 1.22 of Ref.[6]) requires an enlarged phase space with as many new pairs of canonical variables as pairs of second class constraints. The second class constraints are transformed into first class ones by inserting a suitable dependence on the new canonical variables. This method has a great degree of arbitrariness, modifies the theory off-shell and, having new gauge invariances, has to redefine the canonical Hamiltonian and the observables.

\section{The Shanmugadhasan Canonical Transformations for the Identification of the Gauge Variables and of the Physical Dirac Observables}

We have defined a DO as a first class function on phase space restricted to the constraint sub-manifold $\bar{\gamma}$ : this means that it must have weakly zero Poisson bracket with all the first and second class constraints and that, as a consequence, it is constant on the gauge orbits, namely that it is associated with a function on the reduced phase space. Since DO's describe 
the dynamical content of a singular dynamical system, it is important to find an algorithm for the determination of a canonical basis of them to be able to visualize such a content. This would allow to determine all possible Dirac observables of the singular system and would open the path to the attempt to quantize only the dynamical degrees of freedom of the system as an alternative to Dirac quantization with subsequent reduction at the quantum level (see for instance the BRST observables of the BRST quantization [6]).

This strategy is possible due to the class of canonical transformations discovered by Shanmugadhasan [35] studying the reduction to normal form of a canonical differential system like the EL equations of singular Lagrangians. By using the Lie theory of function groups [36], Shanmugadhasan showed that in each neighborhood in $T^{*} Q$ of a point of the constraint sub-manifold $\bar{\gamma}$ there exists local Darboux bases, whose restriction to $\bar{\gamma}$ allows one to separate the gauge variables from a local Darboux basis of DO's . These canonical transformations are implicitly used by Faddeev and Popov to define the measure of the phase space path integral and produce a trivialization of the BRST approach. See also Refs.[37, 38].

Given a $2 n$-dimensional phase space $T^{*} Q$, the set $G$ of all the functions $\phi\left(\bar{F}_{a}\right)$ of $r$ independent functions $\bar{F}_{1}(q, p), . ., \bar{F}_{r}(q, p)$ (the basis of $G$ ) such that $\left\{\bar{F}_{a}, \bar{F}_{b}\right\}=\phi\left(\bar{F}_{c}\right)(a, b, c=1, . ., r)$ is said to be a function group of rank $r$. If $\phi_{1}, \phi_{2} \in G$, then $\left\{\phi_{1}, \phi_{2}\right\} \in G$. When $\left\{\bar{F}_{a}, \bar{F}_{b}\right\}=0$ for the values of $a$ and $b$, the function group $G$ is said commutative. A subset of $G$ which forms a function group is a sub-group of $G$. If two function groups $G_{1}, G_{2}$ of rank $r$ have $\mathrm{p}$ independent functions in common, they are the basis of a sub-group of both $G_{1}$ and $G_{2}$. A function $\phi \in G$ is said singular if it has zero Poisson bracket with all the functions of $G$; the independent singular functions of $G$ form a sub-group. Given a function group $G$ of rank $r$, it can be shown that the system of partial differential equations $\left\{\bar{g}, \bar{F}_{a}\right\}=0$, $a=1, . ., r$, admits $2 n-r$ independent functions $g_{k}, k=1, . ., 2 n-r$, as solutions and they define a reciprocal function group $G^{r}$ of rank $2 n-r$. The basis functions of $G$ and $G^{r}$ are in involution under Poisson brackets.

The following two theorems on function groups and involutory systems [36] are the basis of Shanmugadhasan theory:

i) For a non-commutative function group $G$ of rank $r$ there exists a canonical basis $\bar{\phi}_{1}, .$. , $\bar{\phi}_{m+q}, \bar{\psi}_{1}, \ldots, \bar{\psi}_{m}$ with $2 m+q=r$ such that

$$
\left\{\bar{\phi}_{\lambda}, \bar{\phi}_{\mu}\right\}=\left\{\bar{\psi}_{\alpha}, \bar{\psi}_{\beta}\right\}=0, \quad\left\{\bar{\phi}_{\alpha}, \bar{\psi}_{\lambda}\right\}=\delta_{\alpha \lambda}, \quad \alpha, \beta=1, . ., m, \lambda, \mu=1, . ., m+q .
$$

As a corollary a non-commutative function group $G$ of rank $r$ is a sub-group of a function group of rank $2 n$, whose basis $\bar{\phi}_{1}, . ., \bar{\phi}_{n}, \bar{\psi}_{1}, . ., \bar{\psi}_{n}$ can be chosen so that

$$
\left\{\bar{\phi}_{i}, \bar{\phi}_{j}\right\}=\left\{\bar{\psi}_{i}, \bar{\psi}_{j}\right\}=0, \quad\left\{\bar{\phi}_{i}, \bar{\psi}_{j}\right\}=\delta_{i j}, \quad i, j=1, . ., n .
$$

ii) A system of $2 m+q$ independent equations (defining a surface $\bar{\gamma}$ of dimension $2(n-m)-q$ in $\left.T^{*} Q\right)$

$$
\bar{\Omega}_{a}(q, p)=0, \quad a=1, . ., 2 m+q,
$$

such that $\operatorname{rank}\left\{\bar{\Omega}_{a}, \bar{\Omega}_{b}\right\}=2 m$, can be substituted by a locally equivalent system

$$
\bar{\phi}_{\lambda}(q, p)=0, \quad \lambda=1, . ., m+q, \quad \bar{\psi}_{\alpha}(q, p)=0, \quad \alpha=1, . ., m,
$$

for which the relations 


$$
\left\{\bar{\phi}_{\lambda}, \bar{\phi}_{\mu}\right\}=\left\{\bar{\psi}_{\alpha}, \bar{\psi}_{\beta}\right\}=0, \quad\left\{\bar{\psi}_{\alpha}, \bar{\phi}_{\lambda}\right\}=\delta_{\alpha \lambda}
$$

hold locally in $T^{*} Q$. Therefore Eqs.(4.6) are equivalent to the vanishing of the canonical basis of a non-commutative function group of rank $2 m+q$.

Let us consider a dynamical system with a $n$-dimensional configuration space $Q$ described by a singular Lagrangian, whose associated Hamiltonian description contains: i) a set of first class constraints $\bar{\Phi}_{(1) \mathcal{A}}(q, p) \approx 0, \mathcal{A}=1, . ., M$, among which the primary ones are $\bar{\phi}_{(o) \bar{A}}(q, p) \approx 0, \bar{A}=1, . ., m$; ii) a set of second class constraints $\bar{\Phi}_{(2) \mathcal{A}^{\prime}}(q, p) \approx 0, \mathcal{A}^{\prime}=$ $1, . ., 2 s_{2}$; iii) a final Dirac Hamiltonian $\bar{H}_{D}^{(F)}=\bar{H}_{c}^{(F)}(q, p)+\sum_{\bar{a}} \lambda^{(o) \bar{A}}(t) \bar{\phi}_{\bar{A}}(q, p)$. In the $2 n$-dimensional phase space $T^{*} Q$ the dynamics is restricted to the final $\left[2\left(n-s_{2}\right)-M\right]$ dimensional constraint sub-manifold $\bar{\gamma} \subset \ldots \subset \gamma \subset T^{*} Q(\gamma$ is the primary sub-manifold; if there are only first class constraints $\bar{\gamma}$ is a presymplectic manifold; however the term presymplectic manifold is often used to denote a generic $\bar{\gamma}$ ), whose closed degenerate twoform $\bar{\omega}_{\bar{\gamma}}$ has dimension ker $\bar{\omega}_{\bar{\gamma}}=M$. We have $\bar{\gamma} \subset \gamma_{(2)} \subset T^{*} Q$, where $\gamma_{(2)}$ is the $2\left(n-s_{2}\right)$ dimensional sub-manifold defined by the second class constraints with the symplectic twoform $\bar{\omega}_{(2)}$ giving rise to the Dirac brackets.

Let the constraints constraints form a function group of rank $2 s_{2}+m$.

Theorem ii) ensures that in every neighborhood in $T^{*} Q$ of a point of $\bar{\gamma}$ there exists a passive canonical transformation $\left(q^{i}, p_{i}\right) \mapsto\left(Q^{i}, P_{i}\right)$ in $T^{*} Q$ such that in the new canonical basis the neighborhood is identified by the new constraints

$$
P_{\mathcal{A}} \approx 0, \quad \mathcal{A}=1, . ., m, \quad Q^{a^{\prime}} \approx 0, \quad P_{a^{\prime}} \approx 0, \quad a^{\prime}=1, . ., s_{2} .
$$

Therefore locally we obtain an Abelianization of first class constraints and a canonical form of the second class constraints $\left[Q^{a^{\prime}}=\bar{b}_{\mathcal{A}^{\prime}}{ }^{\prime} \bar{\Phi}_{(2) \mathcal{A}^{\prime}}, P_{a^{\prime}}=\bar{c}_{a^{\prime} \mathcal{A}^{\prime}} \bar{\Phi}_{(2) \mathcal{A}^{\prime}}\right]$ associated with this Abelianization. Eqs.(4.9) give the canonical form of a function group of rank $2 s_{2}+m$. Due to theorem i) the reciprocal function group of rank $2\left(n-s_{2}\right)-m$ has a basis formed by a) $m$ Abelianized gauge variables $Q^{\mathcal{A}}$ parametrizing the $m$-dimensional gauge orbits in $\bar{\gamma}$; b) a canonical basis of Dirac's observables associated with the Abelianization described by the $n-m-s_{2}$ pairs of canonical variables $Q^{a}, P_{a}, a=1, . ., n-m-s_{2}$, which have zero Poisson brackets with the constraints in the form (4.9) by construction. As a consequence they have weakly zero Poisson bracket with all the original constraints, so that they are gauge invariant. This is a local Darboux basis for the presymplectic sub-manifold $\bar{\gamma}$.

Let us remark that the $T^{*} Q$ Poisson bracket $\{., .\}_{Q, P}$ coincides with the Dirac bracket $\{., .\}_{\gamma_{(2)}}^{*}$ when restricted to the second class sub-manifold $\gamma_{(2)}$. Therefore it can be shown $[37,38]$ that the new Dirac Hamiltonian $\bar{H}_{D}^{(F)^{\prime}}\left[p_{i} d q^{i}-\bar{H}_{D}^{(F)} d t=P_{i} d Q^{i}-\bar{H}_{D}^{(F)^{\prime}} d t-d F\right]$ is the first class function

$$
\begin{aligned}
\bar{H}_{D}^{(F)^{\prime}}= & \bar{H}_{c}^{(F)^{\prime}}(Q, P)+\sum_{\bar{a}} \lambda^{(o) \bar{A}}(t) \bar{d}_{\bar{A} \mathcal{B}} P_{\mathcal{B}} \\
\bar{H}_{c}^{(F)^{\prime}}(Q, P)= & \bar{K}_{c}^{(F)}(Q, P)-\tilde{\bar{\Phi}}_{(2) \mathcal{A}^{\prime}}(Q, P) \tilde{\bar{c}}_{\mathcal{A}^{\prime} \mathcal{B}^{\prime}}(Q, P)\left\{\tilde{\bar{\Phi}}_{(2) \mathcal{B}^{\prime}}(Q, P), \bar{K}_{c}^{(F)}(Q, P)\right\} \\
& \tilde{\bar{\Phi}}_{(2) \mathcal{A}^{\prime}}(Q, P)=\bar{\Phi}_{(2) \mathcal{A}^{\prime}}(q(Q, P), p(Q, P)) \\
& \tilde{\bar{c}}_{\mathcal{A}^{\prime} \mathcal{C}^{\prime}}\left\{\tilde{\bar{\Phi}}_{(2) \mathcal{C}^{\prime}}, \tilde{\bar{\Phi}}_{(2) \mathcal{B}^{\prime}}\right\}=\delta_{\mathcal{A}^{\prime} \mathcal{B}^{\prime}} \\
& \left\{\bar{K}_{c}^{(F)}, P_{\mathcal{A}}\right\}=\left\{\bar{K}_{c}^{(F)}, Q^{a^{\prime}}\right\}=\left\{\bar{K}_{c}^{(F)}, P_{a^{\prime}}\right\}=0
\end{aligned}
$$


since we have $\bar{\Phi}_{(1) \mathcal{A}}=\bar{d}_{\mathcal{A B}} P_{\mathcal{B}}+($ terms quadratic in the second class constraints $) \equiv \bar{d}_{\mathcal{A B}} P_{\mathcal{B}}$ near $\bar{\gamma}$.

When we are able to solve the first class constraints $\bar{\Phi}_{(1) \mathcal{A}}(q, p) \approx 0$ in a subset $p_{\mathcal{A}}$ of the momenta, as already said in Subsection IIIC, a possible Abelianized form of the first class constraints is

$$
P_{\mathcal{A}}=p_{\mathcal{A}}-\bar{\psi}_{\mathcal{A}}\left(q^{i}, p_{i \neq \mathcal{B}}\right) \approx 0 .
$$

Therefore with the Shanmugadhasan canonical transformations we are able to separate the gauge degrees of freedom (either inessential variables or, in reparametrization invariant theories, variables describing the generalized inertial effects [4]) from the physical ones at least locally in suitable open sets of $T^{*} Q$ intersecting the constraint sub-manifold $\bar{\gamma}$.

Moreover we see which kind of freedom we have in the choice of the functional form of the primary constraints: at least locally we can always make a choice ensuring the complete diagonalization of chains discussed in Subsection IIID. In a (in general local) Shanmugadhasan basis we have

$$
\begin{aligned}
& \left\{P_{\mathcal{A}}, P_{\mathcal{B}}\right\}=\left\{P_{\mathcal{A}}, Q^{a^{\prime}}\right\}=\left\{P_{\mathcal{A}}, P_{a^{\prime}}\right\}=0, \\
& \left\{Q^{a^{\prime}}, P_{b^{\prime}}\right\}=\delta_{b^{\prime}}^{a^{\prime}}, \quad\left\{Q^{a^{\prime}}, Q^{b^{\prime}}\right\}=\left\{P_{a^{\prime}}, P_{b^{\prime}}\right\}=0, \\
& \left\{\bar{H}_{d}, P_{\mathcal{A}}\right\}=\left\{\bar{H}_{d}, Q^{a^{\prime}}\right\}=\left\{\bar{H}_{d}, P_{a^{\prime}}\right\}=0 .
\end{aligned}
$$

An open fundamental problem is the determination of those singular systems which admit a sub-group of Shanmugadhasan canonical trnsformations globally defined in a neighborhood of the whole constraint sub-manifold $\bar{\gamma}$. When this class of transformations exist, we have a family of privileged canonical bases in which the constraint sub-manifold becomes the direct product of the reduced phase space $\bar{\gamma}_{R}$ (in the simplest case $\bar{\gamma}_{R}=T^{*} Q_{R}$ for some reduced configuration space $Q_{R}$ ) by a manifold $\Gamma$ diffeomorphic to the gauge orbits, $\bar{\gamma}=\bar{\gamma}_{R} \times \Gamma$. When $\bar{\gamma}$ is a stratified sub-manifold, namely it is the disjoint union of different strata $\bar{\gamma}_{a}$ each one with different standard gauge orbit $\Gamma_{a}$ (this may happen if the Hessian matrix has variable rank), the same result may be valid for each stratum, i.e. $\bar{\gamma}_{a}=\bar{\gamma}_{R a} \times \Gamma_{a}$. The existence of privileged canonical bases is a phenomenon induced by the direct product structure and is similar to the existence of special coordinate systems for the separation of variables admitted by special partial differential equations.

In general the topological structure of the original configuration space $Q$ and/or of the constraint sub-manifold $\bar{\gamma} \subset T^{*} Q$ will not allow the existence of this privileged class of canonical transformations. For instance this usually happens when the original configuration space $Q$ is a compact manifold. In these cases the only way to study the constraint submanifold is to use the classical BRST cohomological method [6]. However, even in this cases it is interesting to extrapolate and define new singular dynamical systems with this direct product structure from the local results for the original systems. The study of these new models can give an idea of the non-topological part of the dynamics of the original systems.

Moreover, special relativity induces a stratification of the constraint sub-manifold of relativistic singular systems (all having the Poincare group as the kinematical global Noether symmetry group) according to the types of Poincare' orbit existing for the allowed configurations of the singular isolated system. Again each Poincare' stratum has to be studied separately to see whether it admits the direct product structure. When such a structure 
is present the privileged canonical bases have to be further restricted by selecting the ones whose coordinates are also adapted to the Poincare' group

\section{THE HESSIAN MATRIX OF SINGULAR LAGRANGIANS AND THE EULER-LAGRANGE EQUATIONS}

After the description of the Hamiltonian formalism we analyze the EL equations of singular Lagrangians and their projectability to phase space. Then we give an idea of the pathologies which can appear when the rank of the Hessian matrix is not constant.

\section{A. The Eigenvalues of the Hessian Matrix and the Classification of the Euler- Lagrange Equations.}

After having described the first order Hamiltonian formalism for singular systems, let us come back to the second order formalism based on the singular Lagrangian $L(q, \dot{q})$ and its EL equations. The singular nature of the system is associated with the $m \leq n$ null eigenvalues of the $n \times n$ Hessian matrix $A_{i j}(q, \dot{q})$, $\operatorname{det}\left(A_{i j}(q, \dot{q})\right)=0$. This is the source of the $m$ primary constraints $\bar{\phi}_{A}(q, p) \approx 0, A=1, ., m$, when $\operatorname{rank}\left(A_{i j}(q, \dot{q})\right)=n-m=$ const. everywhere in the $(q, \dot{q})$ space.

Since we have $\phi_{A}(q, \mathcal{P}(q, \dot{q}))=\left.\bar{\phi}_{A}(q, p)\right|_{p=\mathcal{P}(q, \dot{q})} \equiv 0$, we get the identity

$$
0 \equiv \frac{\partial}{\partial \dot{q}^{i}} \phi_{A}(q, \mathcal{P}(q, \dot{q}))=\left.A_{i j}(q, \dot{q}) \frac{\partial \bar{\phi}_{A}}{\partial p_{j}}\right|_{p=\mathcal{P}(q, \dot{q})}
$$

This means that $\left.\frac{\partial \bar{\phi}_{A}}{\partial p_{i}}\right|_{p=\mathcal{P}(q, \dot{q})}$ is a (non-normalized) null eigenvector of the Hessian matrix and that, when the primary constraints are irreducible, each choice of their functional form generates a different basis of $m$ (non-normalized) null eigenvectors for the $m$-dimensional null eigen-space of $A_{i j}(q, \dot{q})$.

If we saturate the EL equations with the null eigenvectors $\left.\frac{\partial \bar{\phi}_{A}}{\partial p_{i}}\right|_{p=\mathcal{P}(q, \dot{q})}$ we get (some of these equations may be void, $0=0$ )

$$
\bar{\chi}_{A}(q, \dot{q})=\left.\left.\frac{\partial \bar{\phi}_{A}}{\partial p_{i}}\right|_{p=\mathcal{P}(q, \dot{q})} L_{i}(q, \dot{q}) \equiv \frac{\partial \bar{\phi}_{A}}{\partial p_{i}}\right|_{p=\mathcal{P}(q, \dot{q})} \alpha_{i}(q, \dot{q}) \stackrel{\circ}{=} 0 .
$$

In the singular case the EL equations are an autonomous system of ordinary differential equations, which cannot be put in normal form and which, in general, contains equations of the second, first and zeroth order as shown by non-void equations (5.2). The first order EL equations are then divided in two groups according to whether they either are or are not projectable to phase space.

i) The zeroth order EL equations are those non-void equations (5.2) which depend only on the configuration coordinates $q^{i}$ 's. They are holonomic Lagrangian constraints. Since we always include among the configuration variables $q^{i}$ eventual (linear or non-linear) Lagrange multipliers, these Lagrangian constraints will appear as secondary Hamiltonian constraints $\bar{\chi}^{(1)}(q) \approx 0$ in $T^{*} Q$ (the primary Hamiltonian constraint being given by the vanishing of the canonical momentum of the Lagrange multiplier).

ii) The non-projectable first order EL equations contained in Eqs.(5.2) are the genuine first order equations of motion. They are also called the primary SODE conditions (see the end of 
Subsection IIB). By using the extended Legendre transformation $\left[g_{(\lambda)}^{A}(q, \dot{q}) \mapsto \lambda^{A}(t)\right.$ for the canonical form of the velocity functions] we get that their Hamiltonian version depends on the Dirac multipliers: in $T^{*} Q$ these equations are recovered from the kinematical half of the Hamilton-Dirac equations on the final constraint sub-manifold $\bar{\gamma}$. These genuine first order equations of motion determine the non-projectable primary (and by induction also the nonprimary) velocity functions associated with the Hamiltonian second class constraints (see the extended second Noether theorem in Section VI). Actually they are the counterpart in the second order formalism of those Hamiltonian equations, like Eq.(3.8), which determine the Dirac multipliers associated with the primary second class constraints (and therefore they determine the velocity functions in canonical form). From Theorems 2 and 3 of Subsection IIID we deduce that the primary SODE conditions correspond to the determination of the Dirac multipliers of pairs of second class 0-chains, while the higher SODE conditions correspond to the determination of the Dirac multipliers for all the second class 1-, 2- .. chains.

iii) The projectable first order EL equations among Eqs.(5.2) are those non-holonomic (also said an-holonomic or integrable) Lagrangian constraints which are projected to the secondary Hamiltonian constraints $\bar{\chi}_{a_{1}}^{(1)}(q, p)$ in $T^{*} Q$.

iv) The remaining combinations of the EL equations, which depend on the accelerations $\ddot{q}^{i}$ in an essential way, are the genuine second order equations of motion.

In addition to the EL equations there are also their consequences, namely all those combinations of the EL equations and of their time derivatives, which do not depend on the accelerations. They will form what is called an invariant system with respect to the EL equations [35] and can be of the types i), ii), iii). The consequences of the EL equations of type ii) are called higher SODE conditions. These aspects of the theory of singular systems will be clarified with the second Noether theorem in Section VI.

Let us remark that the presence of first order EL equations implies that the first order velocity space formalism in the tangent bundle $T Q$ (described in Subsection IIC in the regular case) cannot be extended to singular systems and, as said in Refs.[23-25], till now there is no working formulation but only by hand extensions.

\section{B. Pathologies of Singular Systems with Hessians of Variable Rank}

As shown in Ref [24] (where all the examples quoted in the reported bibliography are analyzed and clarified) when the Hessian matrix has not a constant rank many types of pathologies may appear. To put control on them the basic point is to look for a Hamiltonian formulation of these systems implying that the Euler-Lagrange equations and the Hamilton equations have the same solutions.

The main pathologies are :

a) Third and Fourth Class Constraints.

These new types of non-primary constraints are at the basis of the failure of Dirac's conjecture for many singular Lagrangians with a Hessian matrix of variable rank. This happens because these constraints $\bar{\chi}(q, p) \approx 0$ look like first class constraints. Their associated Hamiltonian vector fields $\bar{X}_{\bar{\chi}}=\{., \bar{\chi}\}$ are either first class, namely tangent to the constraint sub-manifold $\bar{\gamma}$, or vanishing on $\bar{\gamma}$ (but not near $\bar{\gamma}$ ). However, they are not generators of Hamiltonian gauge transformations. Instead, in general they generate spurious solutions of the Jacobi equations, which are not deviations between two neighboring solutions of the EL equations, due to the linearization instability present in these singular systems. As an 
example consider a non-primary constraint $p_{1} \approx 0:$ i) if it is first class, its conjugate variable $q^{1}$ is a gauge variable; ii) if it is second class there is another constraint determining $q^{1}$, so that the pair $q^{1}, p_{1}$ can be eliminated; iii) if it is third class, the conjugate variable $q^{1}$ is determined by one combination of the final Hamilton-Dirac equations and depends on the initial data.

Instead a fourth class or ineffective constraint is a non-primary constraint $\bar{\chi}(q, p) \approx 0$ generated inside a chain by the Dirac algorithm such that $\left.d \bar{\chi}\right|_{\bar{\chi}=0}=0$ even if all the other constraints in the chain have a non-vanishing differential. These constraints have weakly vanishing Poisson brackets with every function on $T^{*} Q$, so that they are first class quantities. For the sake of simplicity, let us consider $p_{1}^{2} \approx 0$ as a non-primary constraint of this type. For the determination of the constraint sub-manifold $\bar{\gamma}$ we must use its linearized form $p_{1} \approx 0$. But we cannot use this linearized form in the final Dirac Hamiltonian generating the final Hamilton-Dirac equations (as instead is usually done), because otherwise the solutions of the Hamilton-Dirac and EL equations do not coincide.

B) Proliferation of Constraints and Ramification of Chains of Constraints.

Let us consider the chains of constraints discussed in Subsection IIID. If in a chain one gets a constraint like $q^{1} q^{2} \approx 0$ (this possible only if the Hessian rank is not constant) then the chain gives rise to three distinct chains (ramification of chains) because the constraint gives rise to the following three sectors: i) $q^{1} \approx 0, q^{2} \approx 0$ (proliferation of constraints); ii) $q^{1} \approx 0, q^{2} \neq 0$; iii) $q^{2} \approx 0, q^{1} \neq 0$.

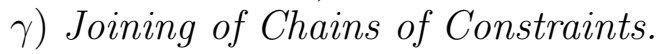

In certain examples after some steps after a ramification of chains there could be a joining of two of the new chains.

Look at Ref. [24] for all the examples of these pathologies and for what is known in mathematical physics on singular systems. Even if we discard all the pathological cases with Hessians of constant rank, there is not a consistent formulation of singular systems covering the second order formalism, the tangent space one and the cotangent Hamiltonian one.

\section{SINGULAR LAGRANGIANS AND THE SECOND NOETHER THEOREM FOR FINITE-DIMENSIONAL SYSTEMS}

After quoting the first Noether theorem and its extensions, we show that behind singular Lagrangians and Hamiltonian constraints there is an extension of the second Noether theorem.

\section{A. Symmetries, the First Noether Theorem and its Extensions.}

The two Noether theorems are a basic ingredient in the study of the consequences of the invariances of Lagrangian systems under continuous symmetry transformations. Ref.[39] gives a review of their applications in theoretical physics, while Ref.[40] contains a review of the intrinsic geometrical formulations and of the various extensions of the first Noether theorem and Refs.[41-43] survey the use of the second theorem. In this Subsection we shall review the first theorem and its extensions. 
For finite-dimensional systems described by a regular (maybe time dependent) Lagrangian $L(t, q, \dot{q})$ the first Noether theorem states that if the action functional $S=\int d t L$ is quasiinvariant under a $r$ parameter group $G_{r}$ of continuous transformations of $t$ and $q^{i}$, then $r$ linear independent combinations of the EL equations $L_{i}$ reduce identically to total time derivatives. The converse is also true under appropriate hypotheses.

This means that if under an infinitesimal set of invertible local variations $\delta_{a} t=\bar{t}_{a}-t=$ $\delta_{a} t(t, q), \delta_{o a} q^{i}=\bar{q}_{a}^{i}(t)-q^{i}(t)=\delta_{o a} q^{i}(t, q, \dot{q})^{3}, a=1, . ., r$, the total variation of $L$ is a total time derivative (the following equation is a Killing-type equation; $\equiv$ means identically )

$$
\begin{aligned}
\delta_{a} L & =L\left(\bar{t}_{a}, \bar{q}_{a}\left(\bar{t}_{a}\right), \frac{d \bar{q}_{a}\left(\bar{t}_{a}\right)}{d \bar{t}_{a}}\right) \frac{d \bar{t}_{a}}{d t}-L(t, q, \dot{q})= \\
& =\frac{\partial L}{\partial q^{i}} \delta_{o a} q^{i}+\frac{\partial L}{\partial \dot{q}^{i}} \delta_{o a} \dot{q}^{i}+\frac{d}{d t}\left(L \delta_{a} t\right)= \\
& =\frac{\partial L}{\partial t} \delta_{a} t+\frac{\partial L}{\partial q^{i}} \delta_{a} q^{i}+\frac{\partial L}{\partial \dot{q}^{i}} \delta_{a} \dot{q}^{i}+L \frac{d \delta_{a} t}{d t}= \\
& =\delta_{o a} q^{i} L_{i}+\frac{d}{d t}\left(\frac{\partial L}{\partial \dot{q}^{i}} \delta_{o a} \dot{q}^{i}+L \delta_{a} t\right) \equiv \frac{d F_{a}(t, q, \dot{q})}{d t}
\end{aligned}
$$

then one obtains the following $r$ Noether identities (both $G_{a}$ and $F_{a}$ are in general functions of $t, q^{i}$ and $\dot{q}^{i}$ )

$$
\begin{aligned}
\frac{d G_{a}}{d t} & \equiv-\delta_{o a} q^{i} L_{i} \stackrel{\circ}{=} 0 \\
G_{a} & =\frac{\partial L}{\partial \dot{q}^{i}} \delta_{o a} q^{i}-F_{a}+L \delta_{a} t=\frac{\partial L}{\partial \dot{q}^{i}} \delta_{a} q^{i}-F_{a}-\left(\dot{q}^{i} \frac{\partial L}{\partial \dot{q}^{i}}-L\right) \delta_{a} t
\end{aligned}
$$

The $r$ quantities $G_{a}(q, \dot{q})$ are constants of the motion (in field theory one would obtain $r$ conservation laws $\left.\partial_{\mu} J_{a}^{\mu} \stackrel{\circ}{=}\right)$. For $F_{a} \neq 0$ we speak of quasi-invariance, while for $F_{a}=0$ of invariance. When we have $\delta_{o a} q^{i}(t, q)$, we get $F_{a}(t, q)$.

It is always possible to define a new set of variations in which $t$ is not varied $\left(\delta_{a}^{\prime} t=0\right.$, $\delta_{o a}^{\prime} q^{i}=\delta_{o a} q^{i}$ ) and which gives rise to the same constants of motion $G_{a}$ : the only difference is that now $\delta_{a}^{\prime} L \equiv \frac{d F_{a}^{\prime}}{d t}$ with $F_{a}^{\prime}=F_{a}-L \delta_{a} t$. In general, there is an infinite family of Noether symmetry transformations $\delta_{a} t, \delta_{o a} q^{i}$ associated with the same set of constants of motion $G_{a}$ (even a change of the functional form of the Lagrangian is allowed: $\delta_{a} L=$ $L^{\prime}$ (barred variables) $-L$ ). See Ref.[40] for a critical review and the proposal of a preferred geometrical approach. Moreover, inside every family of Noether symmetry transformations there are always dynamical symmetry transformations, i.e. symmetry transformations of the EL differential equations mapping the space of its solutions onto itself (the sets of Noether symmetry and dynamical symmetry transformations of a Lagrangian system do not coincide but have an overlap).

The concept of a family of Noether transformations associated with a given set of constants of motion has also been analyzed by Candotti, Palmieri and Vitale [44]. They

${ }^{3}$ The associated global variations (corresponding to Lie derivatives) are $\delta_{a} q^{i}=\bar{q}_{a}^{i}\left(\bar{t}_{a}\right)-q^{i}(t)=\delta_{o a} q^{i}+\dot{q}^{i} \delta_{a} t$. The corresponding variations of the velocities are $\delta_{o a} \dot{q}^{i}=\frac{d}{d t} \delta_{o a} q^{i}, \delta_{a} \dot{q}^{i}=\delta_{o a} \dot{q}^{i}-\dot{q}^{i} \frac{d \delta_{a} t}{d t}$. 
point out that each family contains transformations $\tilde{\delta}_{a} t, \tilde{\delta}_{o a} q^{i}$ such that Eqs.(6.1) become $\tilde{\delta}_{a} L \equiv \frac{d \tilde{F}_{a}}{d t}+f_{a}, \quad f_{a}(t, q, \dot{q}, \ddot{q}) \stackrel{\circ}{=} 0$. That is we have a weak quasi-invariance, because $\delta_{a} L$ only becomes a total time derivative by using the EL equations. The Noether identities (6.2) become $\frac{d \tilde{G}_{a}}{d t} \equiv-\tilde{\delta}_{o a} q^{i} L_{i}+f_{a} \stackrel{\circ}{=} 0$ and give rise to the same constants of motion $G_{a}$, if $\tilde{\delta}_{a} t$, $\tilde{\delta}_{o a} q^{i}, \tilde{F}_{a}$ are such that $\tilde{G}_{a}=\frac{\partial L}{\partial \dot{q}^{i}} \tilde{\delta}_{o a} q^{i}-\tilde{F}_{a}+L \tilde{\delta}_{a} t \stackrel{\circ}{=} G_{a}$. In the regular case these extensions can be considered irrelevant, but it is not so in the singular case.

The generator of the Noether transformation is the vector field $Y=\delta t \frac{\partial}{\partial t}+\delta q^{i} \frac{\partial}{\partial q^{i}}+\delta \dot{q}^{i} \frac{\partial}{\partial \dot{q}^{i}}$ and in terms of it we get $\delta t=Y t, \delta q^{i}=Y q^{i}=\mathcal{L}_{Y} q^{i}, Y L \equiv \dot{F}-L \frac{d \delta t}{d t}$. The constant of motion $G=\frac{\partial L}{\partial \dot{q}^{i}} \delta_{o} q^{i}-F+L \delta t$ is an invariant of the generator $Y: Y G=0$.

The natural setting for the definition and study of the generator $Y$ (and of the dynamical symmetries of differential equations) is the infinite jet bundle [45], where $Y$ is a Lie-Bäcklund vector field ( $Y$ gives its truncation to the first derivatives). As shown in Refs. [45] there are only two kinds of invariance transformations when the number of degrees of freedom is higher than one: i) the Lie point tranformations of $R \times Q$ extended to the higher derivatives; ii) the Lie-Bäcklund transformations (or tangent transformations of infinite order preserving the tangency of infinite order of two curves). These latter have $\delta t$ and/or $\delta_{o} q^{i}$ depending on the velocities and possibly on the higher accelerations. For instance, the non-point canonical transformations of $T^{*} Q$ become Lie-Bäcklund transformations with $\delta t=0$, when rephrased through the inverse Legendre transformation in the second order Lagrangian formalism.

By expressing the velocities in terms of the coordinates and canonical momenta we get $\delta q^{i}(q, \dot{q})=\bar{\delta} \bar{q}^{i}(q, p), F(q, \dot{q})=\bar{F}(q, p), G(q, \dot{q})=\bar{G}(q, p)=p_{i} \overline{\delta q}^{i}(q, p)-\bar{F}(q, p)$. Since the Hamiltonian is defined as $\bar{H}=p_{i} \dot{q}^{i}-L$, the phase space Lagrangian satisfies $\bar{L}(q, p, \dot{q})=$ $p_{i} \dot{q}^{i}-\bar{H}(q, p)=L(q, \dot{q})$ and therefore will have the same invariance properties. This means $\delta \bar{L}=\dot{q}^{i} \overline{\delta p}_{i}+p_{i} \frac{d \delta q^{i}}{d t}-\frac{\partial \bar{H}}{\partial q^{i}} \overline{\delta q^{i}}-\frac{\partial \bar{H}}{\partial p_{i}} \bar{\delta} p_{i}=\overline{\delta p}_{i} \bar{L}_{q i}-\overline{\delta q^{i}} \bar{L}_{p i}+\frac{d}{d t}\left(p_{i} \overline{\delta q^{i}}\right) \equiv \frac{d \bar{F}}{d t}$. Therefore we get $\frac{d}{d t} \bar{G}=\frac{d}{d t}\left(p_{i} \delta q^{i}-\bar{F}\right) \equiv-\bar{\delta}_{i} \bar{L}_{q i}+\overline{\delta q}^{i} \bar{L}_{p i} \stackrel{\circ}{=} 0, \quad \Rightarrow\{\bar{G}, \bar{H}\} \stackrel{\circ}{=} 0$ and $\overline{\delta q^{i}} \equiv \frac{\partial \bar{G}}{\partial p_{i}}=\left\{q^{i}, \bar{G}\right\}$, $\overline{\delta p}_{i} \equiv-\frac{\partial \bar{G}}{\partial q^{i}}=\left\{p_{i}, \bar{G}\right\}$. This is the phase space projected Noether identity associated to the constant of motion $\bar{G}$.

In this way we have found that the Hamiltonian Noether symmetry transformation is generated by the constant of motion $\bar{G}(q, p)(\{\bar{G}, \bar{H}\}=0)$ considered as the generator of a symmetry canonical transformation, i.e. such that the functional form of the Hamiltonian does not change $\left(\delta_{o} H=\bar{H}^{\prime}(q, p)-\bar{H}(q, p)=0\right)$.

The intrinsic formulation of the first Noether theorem and of the reduction of dynamical systems with symmetry, when there is a free and proper symplectic action of a (connected) Lie group on a (connected) symplectic manifold (phase space of an autonomous regular Hamiltonian system with symmetry), is the momentum map approach [46]. For a weakly regular value of the momentum map associated with this action the reduced phase space has a structure of symplectic manifold and inherits a Hamiltonian dynamics. For a singular value of the momentum map the reduced phase space is a stratified symplectic manifold [47]. 


\section{B. The Second Noether Theorem in the Second Order Lagrangian Formalism and its Extension.}

The second Noether theorem states that if the action functional $S=\int d t L$ is quasiinvariant (i.e. its variation is a total time derivative) with respect to an infinite continuous group $G_{\infty r}$, involving up to order $k$ derivatives (i.e. a group whose general transformations depend upon $r$ essential arbitrary functions $\epsilon^{a}(t)$ and their first $k$ time derivatives), then $r$ identities exist among the EL equations $L_{i}$ and their time derivatives up to order $k$. Under appropriate hypotheses the converse also is true.

This version of the theorem is oriented to the description of gauge theories and general relativity, in which there is a singular Lagrangian invariant under local gauge transformations and/or space-time diffeomorphisms and giving rise only to first class constraints at the Hamiltonian level (see for instance Ref.[48]). This is unsatisfactory, because at the Lagrangian level the fundamental property of singular Lagrangians is the number of null eigenvalues of the Hessian matrix and some of them may be associated with Hamiltonian second class constraints (when present). As a consequence in the literature there is no clear statement about the connection between the second Noether theorem and the canonical transformations generated by the constraints when some of them are second class [7]. An extension of the second Noether theorem is needed to include these cases. This was done in Ref.[49] along the lines of the Candotti-Palmieri-Vitale extension [45] of the first Noether theorem using the concept of weak quasi-invariance in the case of a Hessian matrix of constant rank.

The extended second Noether theorem may be expressed by saying that the action functional associated with a singular Lagrangian is weakly quasi-invariant (i.e. quasi-invariant only after having used combinations of the EL equations and of their time derivatives which are independent of the accelerations) under as many sets of local infinitesimal Noether transformations as is the number of null eigenvalues of the Hessian matrix. Each set of such transformations $\delta_{A} q^{i}$ depends on an arbitrary function $\epsilon^{A}(t)$ and its time derivatives up to order $J_{A}$ and produces an identity which can be resolved in $J_{A}+2$ Noether identities, each one being the time derivative of the previous one.

While the $\delta_{A} q^{i}$ 's associated with chains of first class constraints will turn out to be the pull-back by means of the inverse Legendre transformation of the infinitesimal Hamiltonian gauge canonical transformations, the $\delta_{A} q^{i}$ 's associated with chains of second class constraints will turn out to be the pull-back of the infinitesimal canonical transformations generated by the second class constraints (they could be named pseudo-gauge transformations).

This local formulation of the extended theorem which is based on a form of the infinitesimal Noether transformations $\delta_{A} q^{i}$ valid if we use the orthonormal eigenvectors

${ }_{A} \hat{\xi}_{o}^{i}(q, \dot{q})=\tau_{A}^{i}(q, \dot{q})=\left.\frac{\partial \hat{\bar{\phi}}_{A}(q, p)}{\partial p_{i}}\right|_{p=\mathcal{P}(q, \dot{q})}$ of the Hessian matrix. Their use corresponds to the diagonalization of the chains of constraints discussed in Subsection IIID and allows to show that the $J_{A}+2$ Noether identities of the form of Eqs.(6.2) implied by the generalized weak quasi-invariance are projectable to phase space, where they rebuild the whole Dirac algorithm (each chain of identities is connected with a chain in the theorems 2 and 3 of Subsection IIID). See also the next Section. 


\section{CONSTRAINTS IN FIELD THEORIES}

The naive extension of the previous results to classical field theory does not present conceptual problems $[1,5-7,50,51]$. Instead a more rigorous treatment would require much more sophisticated techniques: see for instance Ref.[18] for an introduction to infinite dimensional Hamiltonian systems. The new real phenomenon of field theory with constraints is the possible appearance of the zero modes of the elliptic operators associated with some constraints (due to the spatial gradients of the fields and/or the canonical momenta). It depends on the choice of the function space for the fields, an argument on which there is no general consensus, and creates obstructions to the existence of global gauge fixing constraints like the Gribov ambiguity in Yang-Mills theory (its existence depends upon the choice of the function space [52]).

Let us suppose that we have a singular Lagrangian density $\mathcal{L}\left(\varphi^{r}(x), \varphi_{, \mu}^{r}(x)\right)$ depending upon a set of fields $\varphi^{r}(x), r=1, . ., n$ and their first derivatives $\varphi_{, \mu}^{r}(x)=\partial_{\mu} \varphi^{r}(x)$. The spacetime manifold $M$ of dimension $m+1$ (usually the 4-dimensional Minkowski space-time) has local Cartesian coordinates $x^{\mu}=\left(x^{o}, x^{i}\right)$ and Lorentzian metric $\eta^{\mu \nu}=(1 ;-1, . .,-1)$. The action is the local functional $S=\int d^{m+1} x \mathcal{L}$ and a certain class of boundary conditions at $|\vec{x}| \rightarrow \infty$ for the fields has been chosen in some function space dictated by physical considerations.

With the usual non-covariant choice of $x^{o}$ as time variable we have the following definition of Hessian matrix

$$
A_{r s}(x) \stackrel{\text { def }}{=} A_{r s}^{o o}\left(\varphi^{s}(x), \varphi_{, \mu}^{s}(x)\right)=\frac{\partial^{2} \mathcal{L}}{\partial \varphi_{, o}^{r}(x) \partial \varphi_{, o}^{s}(x)}, \quad A_{r s}^{\mu \nu}=\frac{\partial^{2} \mathcal{L}}{\partial \varphi_{, \mu}^{r} \partial \varphi_{, \nu}^{s}} .
$$

The EL equations implied by Hamilton's principle $\delta S=\delta \int_{\Omega} d^{m+1} x \mathcal{L}=0$ for arbitrary variations $\delta \varphi^{r}(x)$ vanishing on the boundary $\partial \Omega$ of the compact region $\Omega$ are

$$
\begin{gathered}
L_{r}(x)=\frac{\partial \mathcal{L}}{\partial \varphi^{r}(x)}-\partial_{\mu} \frac{\partial \mathcal{L}}{\partial \varphi_{, \mu}^{r}(x)}=-\left(A_{r s}^{\mu \nu}(x) \varphi_{, \nu}^{s}(x)-\alpha_{r}(x)\right)=-\left(A_{r s}(x) \varphi_{, o o}^{s}(x)-\hat{\alpha}_{r}(x)\right) \\
\alpha_{r}-\frac{\partial \mathcal{L}}{\partial \varphi^{r}}-\frac{\partial^{2} \mathcal{L}}{\partial \varphi_{, \mu}^{r} \partial \varphi^{s}} \varphi_{, \mu}^{s}, \quad \hat{\alpha}_{r}=\alpha_{r}-2 A_{r s}^{o i} \varphi_{, o i}^{s}-A_{r s}^{i j} \varphi_{, i j}^{s}
\end{gathered}
$$

The canonical momenta and the standard Poisson brackets (after a suitable definition of functional derivative) are

$$
\pi_{r}(x)=\Pi_{r}^{o}(x), \quad \Pi_{r}^{\mu}(x)=\frac{\partial \mathcal{L}}{\partial \varphi_{, \mu}^{r}(x)}, \quad\left\{\varphi^{r}\left(x^{o}, \vec{x}\right), \pi_{s}\left(x^{o}, \vec{y}\right)\right\}=\delta_{s}^{r} \delta^{m}(\vec{x}-\vec{y}) .
$$

Since the Poisson brackets are local (i.e. they do not depend on primitives of the delta function, a property named local commutativity in Ref.[6]) the Poisson bracket of two local functionals is also a local functional, so that non-local terms cannot be generated through the operation of taking the bracket. For two functions $\bar{F}_{a}\left(\varphi^{r}\left(x^{o}, \vec{x}\right), \varphi_{, i}^{r}\left(x^{o}, \vec{x}\right), \pi_{r}\left(x^{o}, \vec{x}\right), \pi_{r, i}\left(x^{o}, \vec{x}\right)\right), a=1,2$, we have

$$
\left\{\bar{F}_{1}\left(x^{o}, \vec{x}\right), \bar{F}_{2}\left(x^{o}, \vec{y}\right)\right\}=\int d^{m+1} z\left[\frac{\delta \bar{F}_{1}\left(x^{o}, \vec{x}\right)}{\delta \varphi^{r}\left(x^{o}, \vec{z}\right)} \frac{\delta \bar{F}_{2}\left(x^{o}, \vec{x}\right)}{\delta \pi_{r}\left(x^{o}, \vec{z}\right)}-\frac{\delta \bar{F}_{1}\left(x^{o}, \vec{x}\right)}{\delta \pi_{r}\left(x^{o}, \vec{z}\right)} \frac{\delta \bar{F}_{2}\left(x^{o}, \vec{x}\right)}{\delta \varphi^{r}\left(x^{o}, \vec{z}\right)}\right]
$$


Since $\operatorname{det} A_{r s}(x)=0$, there will be a certain number of null eigenvalues of the Hessian matrix with associated local orthonormal null eigenvectors $\tau_{A}^{r}\left(\varphi^{s}(x), \varphi_{, \nu}^{s}(x)\right), A=1, . ., n_{1}$. For the sake of simplicity we shall assume regularity conditions such that the Hessian matrix has a constant rank everywhere.

As in the finite-dimensional case there are $n_{1}$ arbitrary velocity functions non projectable to phase space and $n_{1}$ primary constraints $\bar{\phi}_{A}\left(\varphi^{r}, \varphi_{, i}^{r}, \pi_{r}, \pi_{r, i}\right) \approx 0$ such that $\left.\bar{\phi}_{A}\left(\varphi^{r}, \varphi_{, i}^{r}, \pi_{r}, \pi_{r, i}\right)\right|_{\pi_{r}=\mathcal{P}_{r}(\varphi, \varphi, \mu)} \equiv 0$. Again we have that $\frac{\partial \bar{\phi}_{A}}{\partial \pi_{r}}$ are null eigenvalues of the Hessian matrix. For the sake of simplicity we assume that there is a global functional form of the constraints producing the orthonormal eigenvectors ${ }_{A} \xi_{O}^{i}$.

If the Lagrangian density is sufficiently regular that the Legendre transformation is well defined, the canonical Hamiltonian density is $\overline{\mathcal{H}}_{c}\left(\varphi^{r}(x), \varphi_{, i}^{r}(x), \pi_{r}(x), \pi_{r, i}(x)\right)=$ $\varphi_{, o}^{r}(x) \pi_{r}(x)-\mathcal{L}\left(\varphi(x), \varphi_{, \mu}(x)\right)$, while the Dirac Hamiltonian is

$$
\bar{H}_{D}=\int d^{m} x\left(\overline{\mathcal{H}}_{c}\left(x^{o}, \vec{x}\right)+\sum_{A} \lambda^{A}\left(x^{o}, \vec{x}\right) \bar{\phi}_{A}\left(x^{o}, \vec{x}\right)\right)=\bar{H}_{c}+\sum_{A} \bar{H}_{A} .
$$

Only by choosing consistent boundary conditions for the fields and the Dirac multipliers we can interpret the $\bar{H}_{A}$ 's as generators of local Noether transformations. The Hamilton equations are

$$
\begin{aligned}
\bar{L}_{D \varphi}^{r} & =\varphi_{, o}^{r}\left(x^{o}, \vec{x}\right)-\left\{\varphi^{r}\left(x^{o}, \vec{x}\right), \bar{H}_{D}\right\} \stackrel{\circ}{=} 0, \\
\bar{L}_{D p r} & =p_{r, o}\left(x^{o}, \vec{x}\right)-\left\{p_{r}\left(x^{o}, \vec{x}\right), \bar{H}_{D}\right\} \stackrel{\circ}{=} 0 .
\end{aligned}
$$

Having the primary constraints $\bar{\phi}_{A}\left(x^{o}, \vec{x}\right) \approx 0$ and the Dirac Hamiltonian Dirac's algorithm is plainly extended to field theory starting with the study of the time constancy of the primary constraints. One arrives at a final constraint sub-manifold $\bar{\gamma}$, divides the final set of constraints in first and second class ones and determines the Dirac Hamiltonian of $\bar{\gamma}$ as $\bar{H}_{D}=\bar{H}_{c}+\int d^{m} x \sum_{\bar{A}} \lambda^{\bar{A}}\left(x^{o}, \vec{x}\right) \bar{\phi}_{\bar{A}}\left(x^{o}, \vec{x}\right)$, where $\bar{\phi}_{\bar{A}}\left(x^{o}, \vec{x}\right) \approx 0$ are the first class primary constraints and $\lambda^{\bar{A}}\left(x^{o}, \vec{x}\right)$ the Dirac multipliers, equal to the primary arbitrary velocity functions $g_{(\lambda)}^{\bar{A}}\left(\varphi^{r}\left(x^{o}, \vec{x}\right), \varphi_{, \mu}^{r}\left(x^{o}, \vec{x}\right)\right)$ through the first half of the Hamilton-Dirac equations.

However, besides regularity conditions on the singular Lagrangian density $\mathcal{L}$ so to avoid the (non-explored) field theory counterparts of the pathologies of Subsection VB, one has to consider extra requirements peculiar to field theory:

i) The constraints must define a sub-manifold of the infinite-dimensional phase space, whose properties depend on the choice of the boundary conditions and the function space for the fields and their canonical momenta. This function space must include all physically interesting solutions of the Hamilton-Dirac equations. The constraints must not only be local functionals of the fields but must also be locally complete [6]. This means that every phase space function vanishing on $\bar{\gamma}$ is zero by virtue of the constraints defining $\bar{\gamma}$ and their spatial derivatives of any order only, without having to invoke the boundary conditions. To put mathematical control on BRST cohomology (Ref.[6], theorem 12.4) one needs strong regularity conditions implying that every function vanishing on $\bar{\gamma}$ can be written as combination of the constraints and a finite arbitrary number of their spatial derivatives.

ii) In Ref.[53] it is pointed out that in field theory each constraint $\bar{\phi}\left(x^{o}, \vec{x}\right) \approx 0$ represents a continuous and infinite number of constraints characterized by the space label $\vec{x}$ so that problems may arise with the theory of distributions. Subtle difficulties may appear in the division of the constraints in the first and second class groups and in the mathematical definition of Dirac brackets where the inverse of continuous matrices $C\left(x^{o}, \vec{x}, \vec{y}\right)$ are needed. 
A related problem is with the gauge transformations generated by first class constraints $\bar{\phi}\left(x^{o}, \vec{x}\right) \approx 0$. If we consider the most general generator $\bar{G}=\int d^{m} x \alpha\left(x^{o}, \vec{x}\right) \bar{\phi}\left(x^{o}, \vec{x}\right)$, is $\bar{G}$ a generator of gauge transformations for every parameter function $\alpha\left(x^{o}, \vec{x}\right)$ ? In Ref.[54] the following distinction between proper and improper gauge transformations was given:

a) Proper gauge transformations represent true gauge symmetries of the theory and do not change the physical state of the system. They can be eliminated by fixing the gauge.

b) Improper gauge transformations (they do not exist for finite-dimensional systems) do change the physical state of the system, mapping (on-shell) one physical solution onto a different physical solution. They cannot be eliminated by fixing the gauge but only by means of superselection rules selecting a particular set of solutions.

Given the function space $\mathcal{F}$ for the fields, the problem is the determination of the allowed function space of the parameter functions $\alpha\left(x^{o}, \vec{x}\right)$ so that $\bar{G}$ is the generator of a proper gauge transformation. Not to over-count the constraints the space $\mathcal{F}_{d}$ of the allowed $\alpha\left(x^{o}, \vec{x}\right)$ (the so-called dual space) must be such that, when $\alpha$ varies in this space, $\bar{G} \approx 0$ has an information equivalent to the original constraints $\bar{\phi}\left(x^{o}, \vec{x}\right) \approx 0$. If $\alpha\left(x^{o}, \vec{x}\right)$ does not belong to the dual space, then $\bar{G}$ is the generator of an improper gauge transformation. Under both proper and improper gauge transformations a field belonging to $\mathcal{F}$ must be transformed in a field still in $\mathcal{F}$. Therefore, if $\varphi\left(x^{o}, \vec{x}\right) \in \mathcal{F}$ and $\pi\left(x^{o}, \vec{x}\right) \in \mathcal{F}$ are the fields, then $\delta \varphi\left(x^{o}, \vec{x}\right)=\left\{\varphi\left(x^{o}, \vec{x}\right), \bar{G}\right\}=\frac{\delta \bar{G}}{\delta \pi\left(x^{o}, \vec{x}\right)}$ and $\delta \pi\left(x^{o}, \vec{x}\right)=\left\{\pi\left(x^{o}, \vec{x}\right), \bar{G}\right\}=\frac{\delta \bar{G}}{\delta \varphi\left(x^{o}, \vec{x}\right)}$ must be in $\mathcal{F}$. Moreover the functional derivatives must be well defined, namely we must have $\delta \bar{G}=\int d^{m} x\left(\frac{\delta \bar{G}}{\delta \varphi\left(x^{o}, \vec{x}\right)} \delta \varphi\left(x^{o}, \vec{x}\right)+\frac{\delta \bar{G}}{\delta \pi\left(x^{o}, \vec{x}\right)} \delta \pi\left(x^{o}, \vec{x}\right)\right.$. In general to get this result one has to do a number of integrations by parts and to check whether the resulting surface terms vanish: if they vanish we have a proper gauge transformation with $\alpha\left(x^{o}, \vec{x}\right) \in \mathcal{F}_{d}$. If the $\alpha\left(x^{o}, \vec{x}\right)$ are such that the surface terms do not vanish, we have to modify the generator $\bar{G}$ by adding a surface term, $\bar{G} \mapsto \bar{G}^{\prime}=\bar{G}+\bar{G}_{S T}$, whose variation $\delta \bar{G}_{S T}$ cancels the unwanted surface terms: in this case $\bar{G}^{\prime}$ is the generator of an improper gauge transformation and $\bar{G}^{\prime} \neq 0$ on $\bar{\gamma}$, where it becomes the constant surface term $\bar{G}_{S T}$ commuting with the Hamiltonian. This constant surface term is a non-trivial constant of the motion which can be fixed only with a super-selection rule.

iii) In Ref.[55] it is pointed out that the study of the formal integrability of the partial differential Hamilton equations requires the use of prolongation methods in the infinite jet bundle (namely we have to consider derivatives of the original equations till the needed order) and in particular the determination of a system of equations in involution. While Dirac's algorithm considers all possible consequences of taking the time derivatives of the Hamilton equations, it says nothing about their spatial derivatives. Therefore in field theory one has to check whether extra integrability conditions appear by considering these spatial derivatives.

In the regular case the first Noether theorem implies the existence of conservation laws $\partial_{\mu} G^{\mu}(x) \stackrel{\circ}{=}$, so that with suitable boundary conditions on the fields conserved charges $Q=$ $\int d^{m} x G^{o}\left(x^{o}, \vec{x}\right), \frac{d Q}{d x^{\circ}} \stackrel{\circ}{=} 0$ are obtained.

In the singular case, by using the orthonormal eigenvectors of the Hessian matrix the extended second Noether theorem states that with each null eigenvalue of this matrix is associated a local Noether transformation $\delta_{A} x^{\mu}=0, \delta_{A} \varphi^{r}(x)=\epsilon^{A}(x){ }_{A} \xi_{J_{A}}^{r}(x)+$ $\sum_{j=1}^{J_{A}} \epsilon^{A}{ }_{, \mu_{1} \ldots \mu_{j}}(x){ }_{A} \xi_{J_{A}-j}^{r\left(\mu_{1} \ldots \mu_{j}\right)}(x)\left(\left(\mu_{1} \ldots \mu_{j}\right)\right.$ means symmetrization in the indices), under which we get the following weak quasi-invariance 


$$
\delta_{A} \mathcal{L}=\delta_{A} \varphi^{r} L_{r}+\partial_{\mu}\left(\frac{\partial \mathcal{L}}{\partial \varphi_{, \mu}^{r}} \delta_{A} \varphi^{r}\right) \equiv \partial_{\mu} F_{A}^{\mu}+\epsilon^{A}(x) D_{A} \stackrel{\circ}{=} \partial_{\mu} F_{A}^{\mu} .
$$

Here $F_{A}^{\mu}=F_{A}^{\mu}\left(\varphi, \varphi_{, \nu}, \epsilon^{A}\right)$ and $D_{A}\left(\varphi, \varphi_{, \nu}\right) \stackrel{\circ}{=} 0$ by using the acceleration-independent consequences of the EL equations. By posing

$$
\begin{aligned}
F_{A}^{\mu}\left(\varphi, \varphi_{, \nu}, \epsilon^{A}\right)= & \epsilon^{A}(x){ }_{A} F_{J_{A}}^{\mu}\left(\varphi, \varphi_{, \nu}\right)+\sum_{j=1}^{J_{A}} \epsilon_{, \mu_{1} \ldots \mu_{j}}^{A}(x)_{A} F_{J_{A}-j}^{\mu\left(\mu_{1} \ldots \mu_{j}\right)}\left(\varphi, \varphi_{, \nu}\right) \\
G_{A}^{\mu}\left(\varphi, \varphi_{, \nu}, \epsilon^{A}\right)= & \frac{\partial \mathcal{L}}{\partial \varphi_{, \mu}^{r}} \delta_{A} \varphi^{r}-F_{A}^{\mu}=\epsilon^{A}(x)_{A} G_{J_{A}}^{\mu}+\sum_{j=1}^{J_{A}} \epsilon_{, \mu_{1} \ldots \mu_{j}}^{A}(x)_{A} G_{J_{A}-j}^{\mu\left(\mu_{1} \ldots \mu_{j}\right)}\left(\varphi, \varphi_{, \nu}\right) \\
& { }_{A} G_{J_{A}-j}^{\mu\left(\mu_{1} \ldots \mu_{j}\right)}=\frac{\partial \mathcal{L}}{\partial \varphi_{, \mu}^{r}}{ }_{A} \xi_{J_{A}-j}^{r\left(\mu_{1} \ldots \mu_{j}\right)}-{ }_{A} F_{J_{A}-j}^{\mu\left(\mu_{1} \ldots \mu_{j}\right)}
\end{aligned}
$$

we get the following Noether identities

$$
\partial_{\mu} G_{A}^{\mu} \equiv \epsilon^{A}(x) D_{A}-\delta_{A} \varphi^{r} L_{r} \stackrel{\circ}{=} 0,
$$

which imply

$$
\begin{aligned}
& { }_{A} G_{o}^{\left(\mu_{1}\left(\mu_{2} \ldots \mu_{J_{A}+1}\right)\right)} \equiv 0, \\
& \partial_{\mu A} G_{o}^{\mu\left(\mu_{1} \ldots \mu_{J_{A}}\right)} \equiv-{ }_{A} G_{1}^{\left(\mu_{1}\left(\mu_{2} \ldots \mu_{J_{A}}\right)\right)}-{ }_{A} \xi_{o}^{r\left(\mu_{1} \ldots \mu_{J_{A}}\right)} L_{r}, \\
& \partial_{\mu A} G_{J_{A}-j}^{\mu\left(\mu_{1} \ldots \mu_{j}\right)} \equiv-{ }_{A} G_{J_{A}-j+1}^{\left(\mu_{1}\left(\mu_{2} \ldots \mu_{j}\right)\right)}-{ }_{A} \xi_{J_{A}-j}^{r\left(\mu_{1} \ldots \mu_{j}\right)} L_{r}, \quad j=1, . ., J_{A}-1, \\
& \partial_{\mu{ }_{A}} G_{J_{A}}^{\mu} \equiv D_{A}-{ }_{A} \xi_{J_{A}}^{r} L_{r} .
\end{aligned}
$$

These equations imply the following form of the Noether identities

$$
\begin{aligned}
& \partial_{\mu_{1}} \ldots \partial_{\mu_{J_{A}+1}}{ }_{A} G_{o}^{\left.\mu_{1}\left(\mu_{2} \ldots \mu_{J_{A}+1}\right)\right)} \equiv 0, \\
& \partial_{\mu_{1}} \ldots \partial_{\mu_{j+1}}{ }_{A} G_{J_{A}-j}^{\left.\mu_{1}\left(\mu_{2} \ldots \mu_{j+1}\right)\right)} \equiv \sum_{h=0}^{J_{A}-j-1}(-)^{J_{A}-j-h} \partial_{\mu_{1}} \ldots \partial_{\mu_{J_{A}-h}}\left({ }_{A} \xi_{h}^{r\left(\mu_{1} \ldots \mu_{J_{A}-h}\right)} L_{r}\right) \stackrel{\circ}{=} 0 \\
& \ldots . \quad j=1, . ., J_{A}, \\
& D_{A} \equiv \sum_{h=0}^{J_{A}}(-)^{J_{A}-h} \partial_{\mu_{1}} \ldots \partial_{\mu_{J_{A}-h}}\left({ }_{A} \xi^{r\left(\mu_{1} \ldots \mu_{J_{A}-h}\right)} L_{r}\right) \stackrel{\circ}{=} 0
\end{aligned}
$$

When we have $D_{A} \equiv 0$ (quasi-invariance, first class constraints) we get the contracted Bianchi identities

$$
\sum_{h=0}^{J_{A}}(-)^{J_{A}-h} \partial_{\mu_{1}} \ldots \partial_{\mu_{J_{A}-h}}\left({ }_{A} \xi^{r\left(\mu_{1} \ldots \mu_{J_{A}-h}\right)} L_{r}\right) \equiv 0
$$


We have given a formulation of the theorem based only on the variation of the Lagrangian density. Usually, in absence of second class constraints, namely with $D_{A}(x) \equiv 0$, and with $\delta_{A} \varphi^{r}$ depending only on $\epsilon^{A}(x)$ and $\partial_{\mu} \epsilon^{A}(x)$, one considers the variation of the action evaluated on a compact region $\Omega$ of the $m$-dimensional space bounded by two hyper-planes ( $\Sigma_{f}$ at $x_{f}^{o}$ and $\Sigma_{i}$ at $x_{i}^{o}$; the variations are assumed to vanish on the spatial boundary) and asks for $\delta S=0$. Then the identity (7.9) becomes $\int_{\Omega} d^{m+1} x \delta_{A} \varphi^{r} L_{r} \equiv \int_{\Sigma_{i}} d^{m} \sigma_{\mu} G_{A}^{\mu}\left(x_{i}^{o}, \vec{x}\right)-$ $\int_{\Sigma_{f}} d^{m} \sigma_{\mu} G_{A}^{\mu}\left(x_{f}^{o}, \vec{x}\right)\left[d^{m} \sigma_{\mu}=d^{m} x n_{\mu}\right.$ with $n_{\mu}$ outer normal to the hyper-plane $]$, namely it take the form of a term in the interior of $\Omega$ equated to boundary terms on the hyper-planes. If we ask that the arbitrary functions $\epsilon^{A}(x)$ and their derivatives vanish on the boundary, $\delta_{A} S=0$ (original Noether statement) implies the vanishing of the interior term: $\delta_{A} \varphi^{r} L_{r} \equiv 0$ and this gives the contracted Bianchi identities. By combining this result with the identities one can recover Utiyama [56] and Trautman[57] results, i.e. their form of the identities. For a detailed discussion of this point and of the connected interpretative ambiguities see Refs.[58-60].

If in the Noether identity (7.9) we put $\epsilon^{A}(x)=$ const., this global sub-group of gauge transformations gives rise to the first Noether theorem associated with the Noether transformation $\delta_{A} \varphi^{r}=\epsilon_{A}^{A} \xi_{J_{A}}^{r}$ as a sub-case of the second theorem and to the Noether identity

$$
\partial_{\mu A} G_{J_{A}}^{\mu} \equiv D_{A}-{ }_{A} \xi_{J_{A}}^{r} L_{r} \stackrel{\circ}{=} 0 .
$$

Eqs.(7.13) are the weak conservation laws and the weak (so called) improper conserved (Noether) current is ${ }_{A} G_{J_{A}}^{\mu}$. Instead it can be checked that the strong conservation laws $\partial_{\mu} V_{A}^{\mu} \equiv 0$ hold independently from the EL equations for the following strong improper conserved current (it is not a Noether current)

$$
V_{A}^{\mu}={ }_{A} G_{J_{A}}^{\mu}-\sum_{h=0}^{J_{A}-1}(-)^{J_{A}-h} \partial_{\mu_{1}} \ldots \partial_{\mu_{J_{A}-h-1}}\left({ }_{A} \xi_{h}^{r\left(\mu \mu_{1} \ldots \mu_{J_{A}-h-1}\right)} L_{r}\right)=\partial_{\nu} U_{A}^{[\mu \nu]} \stackrel{\circ}{=}{ }_{A} G_{J_{A}}^{\mu},
$$

where $U_{A}^{[\mu \nu]}([\mu \nu]$ means antisymmetrization) is the following super-potential

$$
U_{A}^{[\mu \nu]}=\sum_{h=1}^{J_{A}-1}(-)^{J_{A}-h} \partial_{\mu_{1}} \ldots \partial_{\mu_{J_{A}-h-1}}\left({ }_{A} G_{h}^{\left(\nu\left(\mu \mu_{1} \ldots \mu_{J_{A}-h-1}\right)\right)}-{ }_{A} G_{h}^{\left(\mu\left(\nu \mu_{1} \ldots \mu_{J_{A}-h-1}\right)\right)}\right) .
$$

The improper strong $Q_{A}^{(S)}$ and weak $Q_{A}^{(W)}$ conserved charges $\left(\frac{d Q_{A}^{(W)}}{d x^{o}} \stackrel{\circ}{=} 0, \frac{d Q_{A}^{(S)}}{d x^{o}} \equiv 0\right.$ for suitable boundary conditions) coincide on the solutions of the acceleration-independent EL equations $[\Omega$ is a spatial volume with boundary $\partial \Omega]$

$$
Q_{A}^{(S)}=\int_{\Omega} d^{m} x V_{A}^{o}\left(x^{o}, \vec{x}\right)=\int_{\partial \Omega} d^{m-1} \Sigma_{k} U_{A}^{[o k]}\left(x^{o}, \vec{x}\right) \stackrel{\circ}{=} Q_{A}^{(W)}=\int_{\Omega} d^{m} x{ }_{A} G_{J_{A}}^{o}\left(x^{o}, \vec{x}\right) .
$$

The source of the ambiguities [58-60] is this doubling of the conserved currents and charges, which does not exist with the first Noether theorem applied to global symmetries.

Finally the generalized Trautman strong conservation laws are (differently from Eqs.(7.9) they hold independently from the EL equations)

$$
\partial_{\mu}\left[G_{A}^{\mu}-\sum_{h=0}^{J_{A}-1} \epsilon_{, \mu_{1} \ldots \mu_{h}}^{A}(x) \sum_{j=h}^{J_{A}}(-)^{j-h} \partial_{\mu_{h+1} \ldots} \partial_{\mu_{j}}\left({ }_{A} \xi_{J_{A}-j}^{r\left(\mu \mu_{1} \ldots \mu_{j}\right)} L_{r}\right)\right] \equiv 0
$$


where for $j=h$ the derivatives acting on the round bracket are absent.

Let us remark (see Ref.[60]) that when a field theory has global symmetry quasiinvariances the first Noether theorem implies the existence of a current which is conserved by using the EL equations: it is the analogue of the weak current, while there is no analogue of the strong current which exists only with gauge symmetries. This gives rise to a conserved charge (the analogue of the weak charge; there is no strong charge in the form of the flux through the surface at infinity of some vector field) and the possibility of a symmetry reduction of the order of the system of equations of motion. Instead in the case of local gauge symmetries we get super-selection rules, not symmetry reduction.

The Noether identities (7.10) and (7.11) have not yet been studied in detail like in the finite-dimensional case, because they contain a lot of information which is not really needed for the Hamiltonian treatment.

\section{FINAL REMARKS}

The problem of quantization of systems with constraints is completely open. The standard approach is the BRST-BV approach for which we send to Refs.[6, 61]. Besides the ordering problem which may create inequivalent quantum systems associated to different orderings of the constraints not quadratic in the canonical variables, there is the problem whether the algebra of quantum constraints based on commutators remains of the same type (first-, second-class or both) as with Poisson brackets.

See Ref.[11] for an approach to quantization oriented to loop quantum gravity and Ref.[62] for the polymer quantization method.

Another ambiguity is whether we quantize all the variables and then we eliminate the gauge ones at the quantum level like it happens with the BRST approach, or whether we eliminate the gauge variables at the classical level and we quantize only the physical degrees of freedom $[25,61]$. The quantization should be independent from the choice of gauge!

In Ref. $[25,63]$ there is a multi-temporal formulation in which every gauge variable is re-interpreted as a "time", with the suggestion that the quantization should be independent from both the ordinary time and the generalized ones. 
[1] P.A.M.Dirac, Generalized Hamiltonian Dynamics , Can.J.Math. 2, 129 (1950); Lectures on Quantum Mechanics, Belfer Graduate School of Science, Monographs Series (Yeshiva University, New York, N.Y., 1964).

[2] J.L.Anderson and P.G.Bergmann, Constraints in Covariant Field Theories, Phys.Rev. 83, 1018 (1951).

[3] P.G.Bergmann and J.Goldberg, Dirac Bracket Transformations in Phase Space, Phys.Rev. 98, 531 (1955).

[4] L.Lusanna, From Clock Synchronization to Dark Matter as a Relativistic Inertial Effect, Lecture at the Black Objects in Supergravity School BOSS2011, Frascati, 9-13 May 2011, Springer Proc.Phys. 144, pp.267-343 (Spinger, Berlin, 2013) (arXiv 1205.2481).

[5] A.J.Hanson, T.Regge and C.Teitelboim, Constrained Hamiltonian Systems, in Contributi del Centro Linceo Interdisciplinare di Scienze Matematiche, Fisiche e loro Applicazioni, n.22 (Accademia Nazionale dei Lincei, Roma, 1975).

[6] M.Henneaux and C.Teitelboim, Quantization of Gauge Systems (Princeton University Press, Princeton, 1992).

[7] K.Sundermeyer, Constraint Dynamics with Applications to Yang-Mills Theory, General Relativity, Classical Spin, Dual String Model, Lecture Notes in Physics, Vol.169 (Springer,Berlin, 1982).

[8] D.M.Gitman and I.V.Tyutin, Quantization of Fields with Constraints (Springer, Berlin, 1990).

[9] J.Govaerts, Hamiltonian Quantization and Constrained Dynamics (Leuwen University Press, Leuwen, 1991).

[10] A.Ashtekar, New Perspectives in Canonical Gravity (Bibliopolis, Naples, 1988); Lectures on Non-Perturbative Canonical Gravity (World Scientific, Singapore, 1991).

[11] T.Thiemann, Modern Canonical Quantum General Relativity (Cambridge Univ.Press, Cambridge, 2007).

[12] M.J.Gotay, J.M.Nester and G.Hinds, Presymplectic Manifolds and the Dirac-Bergmann Theory of Constraints, J.Math.Phys. 19, 2388 (1978)

[13] M.J.Gotay and J.M.Nester, Presymplectic Lagrangian Systems. I : the Constraint Algorithm and the Equivalence Theorem, Ann.Inst.Henri Poincare' A30, 129 (1979) and Presymplectic Lagrangian Systems. II : the Second-Order Equation Problem, A32, 1 (1980).

[14] C.Battle, J.Gomis, J.M.Pons and N.Roma'n-Roy, Equivalence between the Lagrangian and Hamiltonian Formalism for Constrained Systems, J.Math.Phys. 27, 2953 (1986).

[15] A.Echeverri'a-Enri'quez, M.C.Muñoz-Lecanda and N.Roma'n-Roy, Reduction of Presymplectic Manifolds with Symmetry, Rev.Math.Phys. 11, 1209 (1999); A Geometrical Analysis of the Field Equations in Field Theory, Int.J.Math.Meth.Sc. 29, 687 (2002) (arXiv math-ph/0105018); Geometry of Multisymplectic Hamiltonian First-Order Field Theoies, J.Math.Phys. 41, 7402 (2000), (arXiv math-ph/0004005).

[16] M.deLe'on, J.Mari'n-Solano, J.C.Marrero, M.C.Mñoz-Lecanda and N.Roma'n-Roy, Singular Lagrangian Systems on Jet Bundles, Fortsch.Phys. 50, 105 (2002).

[17] H.Cendra, M.Etchechoury and S.J.Ferraro, An Extension of the Dirac and Gotay-Nester Theories of Constraints for Dirac Dynamical Systems, J.Geom.Mech. 6, 167 (2014) (arXiv 1106.3354).

[18] R.Schmidt, Infinite Dimensional Hamiltonian Systems (Bibliopolis, Napoli, 1987). 
[19] F.Gantmacher, Lectures in Analytical Mechanics (Mir, Moscow, 1970).

[20] V.I.Arnold, Mathematical Methods of Classical Mechanics (Springer, New York, 1978).

[21] P.Liebermann and C.M.Marle, Symplectic Geometry and Analytical Mechanics (Reidel, Dordrecht, 1987).

[22] M.Nakahara, Geometry, Topology and Physics (Institute of Physics Publishing, Bristol, 1990)

[23] L.Lusanna, An Enlarged Phase Space for Finite-Dimensional Constrained Systems, Unifying Their Lagrangian,, Phase- and Velocity-Space Descriptions, Phys.Report 185, 1 (1990).

[24] L.Lusanna, The Second Noether Theorem as the Basis of the theory of Singular Lagrangians and Hamiltonian Constraints, Riv.Nuovo Cimento (3)14, 1 (1991).

[25] L.Lusanna, The Shanmugadhasan Canonical Transformation, Function Groups and the Second Noether Theorem, Int.J.Mod.Phys. A8, 4193 (1993); The Relevance of Canonical Transformations in Gauge Theories and General Relativity, Lecture Notes of "Seminario Interdisciplinare di Matematica" (Basilicata Univ.) 5, 125 (2006); Classical Observables of Gauge Theories from the Multitemporal Approach, Contemp.Math. 132, 531 (1992).

[26] F.Loran, Non-Abelianizable First Class Constraints, Commun.Math.Phys. 254, 167 (2005) (arXiv hep-th/0303014).

[27] A.Cabo and D.L.Martinez, On Dirac's Conjecture for Hamiltonian Systems with First- and Second-Class Constraints, Phys. Rev. 42, 2726 (1990).

[28] M.Chaichian, D.Louis Martinez and L.Lusanna, Dirac's Constrained Systems: the Classification of Second-Class Constraints, Ann.Phys. (N.Y.) 232, 40 (1994).

[29] Y.L.Wang, Z.X.Wu, H.Z.Pan, W.T.Lu, H.Jiang and L.Chen, The Limit of Noether Conserved Charges is the Number of Primary First-Class Constraints in a Constrained System, Commun.Theor.Phys. 58, 539 (2012).

[30] Y.L.Wang, C.T.Xu, H.Jiang, W.T.Lu and H.Z.Pan, The Dirac Conjecture and the NonUniqueness of Lagrangian, preprint 2013 (arXiv 1306.3580).

[31] R.Sugano, Y.Kagraoka and T.Kimura, Gauge Transformations and Gauge-Fixing. Condition in Constraint System, Int.J.Mod.Phys. 7, 61 (1992).

[32] L.D.Faddeev and S.L.Shatashvili, Realization of the Schwinger term in the Gauss law and the possibility of correct quantization of a theory with anomalies, Phys.Lett. B167, 225 (1986).

[33] I.A.Batalin and E.S.Fradkin, Operator quantization of dynamical systems with irreducible firstand second-class constraints, Phys.Lett. B180, 157 (1986); Operational quantization of dynamical systems subject to second class constraints, Nucl.Phys. B279, 514 (1987).

[34] I.A.Batalin and I.V.Tyutin, Existence Theorem for the Effective Gauge Algebra in the Generalized Canonical Formalism with Abelian Conversion of Second-Class Constraints, Int.J.Mod.Phys. A6, 3255 (1991).

[35] S.Shanmugadhasan, Canonical Formalism for Degenerate Lagrangians, J.Math.Phys. 14, 677 (1973).

[36] R.O.Fulp and J.A.Marlin, Function groups associated with constraint submanifolds, Rep.Math.Phys. 18, 295 (1980).

[37] L.Castellani, D.Dominici and G.Longhi, Canonical Transformations and Quantization of Singular Lagrangian Systems, Nuovo Cimento 48A, 91 (1978).

[38] D.Dominici and J.Gomis, PoincarCartan Integral Invariant and Canonical Transformations for Singular Lagrangians, J.Math.Phys. 21, 2124 (1980).

[39] B.M.Barbashov and V.V.Nesterenko, Continuous Symmetries in Field Theory, Fortschr.Phys. 31, 535 (1983).

[40] W.Sarlet and F.Cantrijn, Symmetries and Conservation Laws for Generalized Hamiltonian 
Systems, SIAM Rev. 23, 467 (1981).

[41] J.D.Logan, Invariant Variational Principles (Academic Press, New York, N.Y., 1977).

[42] N.P.Konopleva and V.N.Popov, Gauge Fields (Harwood, New York, N.Y., 1981).

[43] J.F.Cariñena, J.A.La'zaro-Cami' and E.Marti'nez, On Second Noether's Theorem and Gauge Symmetries in Mechanics, Int.J.Geom.Meth.Mod.Phys. 3, 471 (2006) (arXiv math/0511180).

[44] E.Candotti, C.Palmieri and B.Vitale, On the Inversion of Noether's Theorem in the Lagrangian Formalism, Nuovo Cimento 70, 233 (1970); Universal Noether's Nature of Infinitesimal Transformations in Lorentz Covariant Field Theories., Nuovo Cimento A7, 271 (1972).

[45] R.L.Anderson and N.H.Ibragimov, Lie-Bäcklund Transformations in Applications (SIAM, Philadelphia, 1979).

[46] J.E.Marsden and A.Weinstein, Reduction of Symplectic Manifolds with Symmetry, Rep.Math.Phys. 5, 121 (1974).

[47] R.Sjamaar and E.Lerman, Stratified Symplectic Spaces and Reduction, Ann.Math. 134, 375 (1991).

[48] B.M.Barbashov and V.V.Nesterenko, Continuous Symmetries in Field Theory, Fortschr.Phys. 31, 535 (1983).

[49] L.Lusanna, An Extension of the Second Noether Theorem, Nuovo Cimento B52, 141 (1979).

[50] P.G.Bergmann, Non-Linear Field Theories, Phys.Rev. 75, 680 (1949).

[51] A.Komar, Field Theoretic Constraint Formalism, Found.Phys. 15, 473 (1985).

[52] V.Moncrief, Gribov Degenracies: Coulomb Gauge Conditions and Initial Value Constraints , J.Math.Phys. 20, 579 (1979).

[53] P.J.Steinhardt, Problems of Quantization in the Infinite Momentum Frame, Ann.Phys. (N.Y.) 128, 425 (1980).

[54] R.Benguria, P.Cordero and C.Teitelboim, Aspects of the Hamiltonian Dynamics of Interacting Gravitational Gauge and Higgs Fields with Applications to Spherical Symmetry, Nucl.Phys. B122, 61 (1977).

[55] W.M.Seiler and R.W.Tucker, Involution and Constrained Dynamics I: the Dirac Approach, J.Phys. A28, 4431 (1995).

[56] R.Utiyama, Invariant Theoretical Interpretation of Interaction, Phys.Rev. 101, 1597 (1956); Theory of Invariant Variation and the Generalized Canonical Dynamics, Prog.Theor.Phys.Suppl. 9, 19 (1959).

[57] A.Trautman, in Gravitation, ed. L.Witten (Wiley, New York, 1962); in Lectures on General Relativity, Brandeis Summer Institute in Theoretical Physics (Prentice Hall, Englewood Cliffs, 1964).

[58] D.L.Karatas and K.L.Kowalski, Noether's Theorem for Local Gauge Transformations, Am.J.Phys. 58, 123 (1990).

[59] H.A.Al-Kuwari and M.O.Taha, Noether Theorem and Local Gauge Invariance, Am.J.Phys. 59, 363 (1991).

[60] K.Brading and H.R.Brown, Noether's Theorems and Gauge Symmetries, preprint 2000, (arXiv hep-th/0009058).

[61] L.Lusanna, On the BRS's, J.Math.Phys. 31, 428 (1990).

[62] B.Dittrich, P.A.Hohn, T.A.Koslowski and M.I.Nelson, Chaos, Dirac Observables and Constraint Quantization, preprint 2015 (arXiv 1508.01947).

[63] L.Lusanna, Lagrangian and Hamiltonian Many-Times Equations, J.Math.Phys. 31, 2126 (1990); Classical Observables of Gauge Theories from the Multitemporal Approach, Contemp.Math. 132, 531 (1992); From Relativistic Mechanics towards Green's Functions: Mul- 
titemporal Dynamics, Proc. VII Seminar on Problems of High Energy Physics and Quantum Field Theory, Protvino 1984 (Protvino University Press, Protvino, 1984). 